\title{
Uso de dispositivos móviles en las aulas de la universidad y rendimiento académico: revisión de la literatura y nueva evidencia en España
}

\author{
Ana Belén Alonso-Conde \\ Departamento de Economía de la Empresa de la Universidad Rey Juan Carlos (Madrid, España) \\ ana.alonso@urjc.es | https://orcid.org/0000-0001-8333-2788 \\ Javier Rojo-Suárez \\ Departamento de Economía de la Empresa de la Universidad Rey Juan Carlos (Madrid, España) \\ javier.rojo@urjc.es | https://orcid.org/0000-0002-7371-3154 \\ José Ángel Zúñiga-Vicente \\ Departamento de Economía de la Empresa (ADO), Economía Aplicada ll y Fundamentos \\ Análisis Económico de la Universidad Rey Juan Carlos (Madrid, España) \\ joseangel.zuniga@urjc.es | https://orcid.org/0000-0002-6783-8348
}

\section{Extracto}

Este trabajo se hace eco de un animado debate, que está presente en los últimos años en el ámbito de la educación superior, sobre si hay que permitir o se debe prohibir el uso de dispositivos móviles por los estudiantes en el aula. A fin de facilitar una toma de decisiones más informada, se revisa en profundidad la evidencia empírica existente hasta la fecha sobre la relación entre el uso de dispositivos móviles en las aulas y el rendimiento académico de los estudiantes. Se proporciona también nueva evidencia empírica sobre dicha relación, considerando ciertos aspectos que han sido ignorados por la investigación previa, como son la asignatura que se enseña, las características/intereses de los docentes, el año de estudio o el tamaño de la clase. Los resultados dispares del análisis empírico realizado, así como de la evidencia disponible, sugieren que el uso de estos dispositivos móviles y su relación con el rendimiento académico es un asunto complejo que debe ser analizado cuidadosamente. Se sugiere que la cuestión de si el uso de dispositivos móviles debe permitirse o prohibirse en el aula debería reemplazarse por cómo los docentes pueden sacar el mejor partido posible del uso de tales dispositivos en sus clases. Además, se presentan otras propuestas que pueden ser especialmente útiles en un contexto educativo como el actual, que está muy condicionado por la crisis de la COVID-19.

Palabras clave: dispositivos móviles; smartphones; ordenadores portátiles; tablets; rendimiento académico; educación superior.

Fecha de entrada: 16-04-2021 / Fecha de revisión: 31-05-2021 / Fecha de aceptación: 02-06-2021

Cómo citar: Alonso-Conde, A. B., Rojo-Suárez, J. y Zúñiga-Vicente, J. Á. (2021). Uso de dispositivos móviles en las aulas de la universidad y rendimiento académico: revisión de la literatura y nueva evidencia en España. Tecnología, Ciencia y Educación, 20, 7-48. https://doi.org/10.51302/tce.2021.600 


\title{
The use of mobile devices in university classrooms and academic performance: literature review and new evidence in Spain
}

\author{
Ana Belén Alonso-Conde \\ Javier Rojo-Suárez \\ José Ángel Zúñiga-Vicente
}

\begin{abstract}
This study echoes a lively debate, which has been present in recent years in the field of higher education about whether to allow or prohibit the use of mobile devices by students in the classroom. In order to facilitate more informed decision-making, the empirical evidence to date on the relationship between the use of mobile devices in classrooms and student academic performance is thoroughly reviewed. New empirical evidence on this relationship is also provided considering certain aspects that have been ignored by past research, such as the type of subject(s), professor's interest/characteristics, the year of study, and even class size. The mixed results obtained from the empirical analysis, as well as the available evidence, suggest that the use of these mobile devices and their relationship with academic performance is a complex issue that need to be carefully examined. We suggest that the question of whether the use of mobile devices should be allowed or prohibited in the classroom should be replaced by how professors can make the best use of these devices in their classes. In addition, other proposals are presented that may be especially useful in an educational context such as the current one, which is highly conditioned by the COVID-19 crisis.
\end{abstract}

Keywords: mobile devices; smartphones; laptops; tablets; academic performance; higher education.

Citation: Alonso-Conde, A. B., Rojo-Suárez, J. and Zúñiga-Vicente, J. Á. (2021). The use of mobile devices in university classrooms and academic performance: literature review and new evidence in Spain. Tecnología, Ciencia y Educación, 20, 7-48. https://doi.org/10.51302/tce.2021.600 


\section{Sumario}

1. Introducción

2. Revisión de la literatura empírica

2.1. Principales ventajas e inconvenientes asociados con el uso de los dispositivos móviles en las aulas universitarias

2.2. Estudios empíricos sobre la relación entre el uso de dispositivos móviles en las aulas universitarias y el rendimiento académico de los estudiantes

3. Metodología

4. Resultados

5. Conclusiones y algunas propuestas para la reflexión

Referencias bibliográficas

Anexo. Investigaciones empíricas sobre la relación entre el uso de los dispositivos móviles en el aula y el rendimiento académico de los estudiantes universitarios 


\section{Introducción}

Los dispositivos móviles, como ordenadores portátiles, tablets y smartphones, se han convertido en un elemento habitual del entorno educativo actual. En las últimas dos décadas, estos dispositivos se han ido incorporando de manera gradual en las distintas etapas del sistema educativo. La introducción de las nuevas tecnologías móviles ha sido promovida por las autoridades públicas. Los Gobiernos de todo el mundo y también numerosas organizaciones internacionales (por ejemplo, la Comisión Europea, la Organización para la Cooperación y el Desarrollo Económicos [OCDE], el Banco Mundial o The United Nations Educational, Scientific and Cultural Organization [UNESCO]) han respaldado la integración de las nuevas tecnologías de la información y la comunicación (TIC) en el sistema educativo como un medio para mejorar los resultados de los estudiantes. Algunos investigadores (por ejemplo, Brown et al., 1998; Brown y Petitto, 2003; Crook y Barrowcliff, 2001) han acuñado el término «computación omnipresente» para describir un campus o centro educativo donde todos o la mayoría de los estudiantes tienen algún tipo de dispositivo móvil y todos los edificios tienen o proporcionan acceso a la tecnología wifi.

La introducción de los distintos tipos de dispositivos móviles en las aulas de los centros educativos se ha justificado porque se supone que pueden ser una herramienta de ayuda para lograr un aprendizaje más eficaz, lo que debería redundar, en última instancia, en un mejor rendimiento académico de los estudiantes de ambos sexos. En el ámbito universitario, la evidencia anecdótica refleja que un número cada vez mayor de docentes está prohibiendo el uso de estos dispositivos en sus clases: se están dando cuenta de que distraen y empeoran significativamente el resultado académico de los estudiantes. Sin embargo, esta reacción adversa hacia la utilización de estos dispositivos en las clases parece que, en un primer momento, ha encontrado más repercusión en los medios de comunicación que dentro del propio mundo académico en lo que a nivel de investigación se refiere. Además, la evidencia contra el uso de estos dispositivos ha sido con frecuencia anecdótica y, probablemente, subjetiva, basada en observaciones de los propios docentes. En cualquier caso, en el debate actual sobre la conveniencia o no de usar estos dispositivos en el aula, los distintos agentes implicados parecen estar cada vez más polarizados en una posición u otra.
En el debate actual sobre la conveniencia o no de usar los dispositivos móviles en el aula, los distintos agentes implicados parecen estar cada vez más polarizados en una posición u otra

Por tanto, es un buen momento para plantearse qué dice la investigación empírica existente sobre el impacto vinculado con el uso de estos dispositivos móviles en el rendimiento 
académico de los estudiantes universitarios. Ciertamente, puede afirmarse que un número cada vez mayor de investigadores ha mostrado un especial interés en indagar en torno a esta importante cuestión con el propósito de ofrecer evidencia estadística o científica rigurosa que vaya más allá de meras opiniones subjetivas. Con ello se pretende facilitar una toma de decisiones mucho más informada a la hora de tener que decidir si prohibir su uso en clase o seguir permitiéndolo (incluso con ciertas restricciones), dadas las importantes implicaciones que podrían derivarse de los resultados obtenidos sobre el propio sistema educativo y, en último término, sobre la sociedad en su conjunto.

Incluso teniendo en cuenta que la investigación realizada hasta el momento utiliza variados enfoques metodológicos y se ha llevado a cabo en distintos contextos de educación superior, los principales hallazgos se pueden resumir de la siguiente manera: por una parte, hay estudios que han encontrado un impacto (correlación/efecto) positivo y significativo derivado del uso de diferentes dispositivos móviles en el aula y el rendimiento académico de los estudiantes universitarios (por ejemplo, Carle et al., 2009; Enríquez, 2010; Karamti, 2016; Kolar et al., 2002; Qi, 2019). Por el contrario, muchos otros estudios encuentran un impacto (correlación/efecto) negativo y significativo (por ejemplo, Carter et al., 2017; Fried, 2008; Glass y Kang, 2019; Grace-Martin y Gay, 2001; Kraushaar y Novak, 2010; LeyrerJackson y Wilson, 2018; Patterson y Patterson, 2017; Sana et al., 2013; Uzun y Kilis, 2019; Zhang, 2015). Finalmente, también hay estudios que muestran que el uso de los distintos dispositivos móviles no está significativamente relacionado con el rendimiento académico o incluso han obtenidos resultados ambiguos (por ejemplo, Hutcheon et al., 2019; Le Roux y Parry, 2017; Parry et al., 2019; Pemberton et al., 2006; Ravizza et al., 2017; Wurst et al., 2008). Por tanto, a la luz de los hallazgos previos, parece claro que aún se requieren más investigaciones para mejorar nuestro conocimiento de este fenómeno.

Con este trabajo se contribuye a este animado debate examinando si existen diferencias significativas en el rendimiento académico entre el grupo de estudiantes universitarios que usan habitualmente dispositivos móviles en el aula y el grupo que nunca los emplea. Sin embargo, en marcado contraste con la mayoría de los estudios previos, los estudiantes analizados se distribuyen en tres clases diferentes: en cada clase hay un grupo de estudiantes de ambos sexos que usan habitualmente ordenadores portátiles y tablets y otro que nunca hace uso de tales dispositivos. Otra nota distintiva es que estos estudiantes han tenido tres docentes diferentes y también estaban en distintos años de estudio. Además, han cursado diferentes materias. En concreto, mediante este trabajo se busca dar una respuesta a las siguientes dos preguntas relacionadas:

- Pregunta 1. ¿Existen diferencias significativas en el rendimiento académico entre el grupo de estudiantes universitarios que utiliza con frecuencia ordenadores portátiles o tablets durante la clase y el grupo de estudiantes que nunca los usa en clase?

- Pregunta 2. ¿Las diferencias significativas en el rendimiento académico se producen independientemente de la materia que cursan los estudiantes, de los docentes que imparten la clase y/o del año de estudio en que se encuentran? 
Este estudio amplía la investigación existente de varias maneras. En primer lugar, es uno de los primeros trabajos en ofrecer una revisión exhaustiva de la literatura sobre la relación entre el uso de dispositivos móviles en el aula (incluyendo ordenadores portátiles, tablets y smartphones) y el rendimiento académico de los estudiantes en contextos de educación superior. Concretamente, se lleva a cabo una revisión de la evidencia empírica durante dos décadas: desde 2000 hasta 2020. Esta revisión será útil para conocer, como punto de partida, si dicha evidencia es unánime y, en caso de ser así, en qué sentido; esto es, si la evidencia existente corrobora que los dispositivos móviles son en realidad una herramienta que puede contribuir de manera significativa a mejorar el rendimiento académico de los estudiantes universitarios o, por contra, lo empeora. Ello ayudará también a tener información más precisa a la hora de valorar hasta qué punto los dispositivos móviles se deben prohibir o no en clase, o si es un buen momento para pensar en replantear esta cuestión.

En segundo lugar, este es uno de los pocos estudios que proporciona información sobre la importancia potencial que puede tener la asignatura que se enseña, las características/intereses del docente y el curso en el que se encuentran los estudiantes. Hasta donde conocemos, solo unas pocas investigaciones (por ejemplo, Gaudreau et al., 2014; Grace-Martin y Gay, 2001; Le Roux y Parry, 2017; Pemberton et al., 2006) han controlado por separado tales aspectos. En este sentido, como argumentan estos y otros investigadores (véase también Kolar et al., 2002), asumimos que la eficacia del uso de dispositivos móviles durante la clase también puede ser función de la materia que se enseña, de las características/ intereses del docente y del curso académico de estudio dentro de la universidad. Sin lugar a dudas, si controlamos todos estos aspectos, podríamos tener, al menos a priori, una imagen bastante más completa o realista del fenómeno de estudio.
Asumimos que la eficacia del uso de dispositivos móviles durante la clase también puede ser función de la materia que se enseña, de las características/intereses del docente y del curso académico de estudio dentro de la universidad

En tercer lugar, la mayoría de las investigaciones previas se han realizado en universidades de EE.UU., Australia, Canadá, Reino Unido o Sudáfrica (es decir, universidades de naturaleza anglosajona que tienen muchas similitudes). Algunos investigadores han sugerido que los valores culturales de un país pueden afectar significativamente al uso de las TIC (véase, por ejemplo, Shuter et al., 2018). Por tanto, es conveniente realizar investigaciones adicionales en contextos universitarios de otros países para determinar si los hallazgos de los distintos trabajos son también generalizables o extrapolables. Para ello, utilizamos una muestra de estudiantes de un grado relacionado con la administración de empresas de una de las universidades públicas más grandes de España. Merece la pena mencionar que esta universidad está firmemente comprometida con el uso de las nuevas TIC. Cabe destacar también que hemos observado una escasez de estudios sobre la cuestión analizada en universidades españolas. En este sentido, consideramos que es importante saber qué sucede en las universidades españolas porque, por ejemplo, según los datos de la OCDE, la tasa de graduación para un título de grado o formación equivalente en España es inferior 
$(34,20 \%)$ al promedio de la OCDE (38,70\%). Específicamente, la tasa de graduación en el campo de la economía, la administración de empresas y el derecho en España es notablemente inferior a la media de la OCDE (18,90\% y 24,20\%, respectivamente) (OCDE, 2020). Así pues, cobra especial relevancia entender cómo puede estar afectando el uso de estos dispositivos en el aula sobre el rendimiento académico de los estudiantes universitarios españoles para poder entender si la diferencia observada en las tasas de graduación puede ser también atribuible a un uso más o menos eficaz de las TIC en las aulas.

Por último, pensamos que el momento actual es especialmente propicio para tratar un tema como este, ya que, como consecuencia de la pandemia de la COVID-19, las clases presenciales se han visto reemplazadas por clases virtuales. Toda la comunidad educativa en general, y la universitaria en particular, se ha visto obligada a hacer un uso inequívoco de las nuevas tecnologías que, por otro lado, en la mayoría de las ocasiones los estudiantes ya venían utilizando normalmente en las clases presenciales. Desde este punto de vista, puede ser interesante, de cara al futuro, comparar los resultados de los estudios realizados hasta la fecha, que toman como base el uso de los dispositivos móviles de manera presencial en las aulas, con los que se deriven, fruto de la nueva situación que estamos viviendo a raíz de la pandemia. Precisamente, esta situación está forzando a los estudiantes a hacer uso de tales dispositivos en sus casas para completar sus procesos de aprendizaje y sacar adelante las respectivas materias.

El resto del trabajo se estructura del siguiente modo. En el segundo apartado se revisa la evidencia existente acerca de la relación entre el uso de los dispositivos móviles en clase y el rendimiento académico de los estudiantes universitarios. En el tercer apartado se presenta la metodología seguida. A continuación, se muestran los resultados obtenidos del análisis empírico realizado. El trabajo culmina con la discusión e implicaciones de los resultados obtenidos, así como con unas propuestas y/o reflexiones finales que pensamos pueden resultar de utilidad para los diferentes grupos de agentes con alguna responsabilidad en el ámbito de la educación universitaria.

\section{Revisión de la literatura empírica}

\subsection{Principales ventajas e inconvenientes asociados con el uso de los dispositivos móviles en las aulas universitarias}

La evidencia muestra que la presencia de diferentes dispositivos móviles, como ordenadores portátiles, tablets o smartphones, en las aulas universitarias ha aumentado de manera muy significativa en los últimos 15 años. Varias razones explican esta circunstancia. Primero, la generación actual de estudiantes universitarios ha crecido inmersa en las nuevas TIC. De hecho, se utilizan habitualmente términos como «nativos digitales», «estudiantes 
digitales», «estudiantes de la era digital» o «generación N», entre otros muchos, para referirse a los estudiantes actuales. Los estudiantes universitarios esperan tener acceso continuo a una amplia variedad de herramientas tecnológicas, y los ordenadores portátiles y las tablets, en un primer momento, y los smartphones, últimamente, se han convertido en la principal opción para el seguimiento de la mayoría de sus asignaturas (Kay y Lauricella, 2014; Montgomery, 2009; Palfrey y Gasser, 2008; Tapscott, 2008).

En segundo lugar, el precio de la mayoría de los dispositivos móviles ha bajado lo suficiente como para que la mayoría de los estudiantes puedan disponer de uno de estos dispositivos sin tener que incurrir en un coste excesivo, y la gran mayoría de las universidades conceden becas y facilitan estos dispositivos a aquellas personas que no tienen acceso a ellos, especialmente durante la pandemia. Según Pearson Student Mobile Device Survey 2015 (Poll, 2015) -una encuesta realizada a una muestra representativa de la población de estudiantes universitarios de ambos sexos en EE. UU. para comprender mejor cómo los estudiantes universitarios usan los dispositivos móviles para el aprendizaje-, el mercado de dichos dispositivos ha crecido significativamente en los últimos años. Esta encuesta también reveló que los ordenadores portátiles seguían siendo el dispositivo móvil más utilizado en clase por los estudiantes universitarios. De hecho, según esta encuesta, alrededor del $87 \%$ de los estudiantes universitarios de EE. UU. usaban un ordenador portátil para el seguimiento de sus clases. En comparación, aproximadamente el $64 \%$ utilizaban un smartphone y el $40 \%$ una tablet, mientras que el $15 \%$ usaba un dispositivo híbrido. En el caso español, según un estudio reciente de Martínez Rodrigo et al. (2019), el ordenador portátil es el equipo preferido por el alumnado en las clases; lo emplea el $87 \%$ para tomar apuntes o seguir las presentaciones en PowerPoint. El teléfono móvil es utilizado por el $75 \%$ de los estudiantes universitarios españoles para realizar consultas puntuales. La tablet es el dispositivo menos utilizado, ya que tan solo el $50 \%$ lo emplea en el aula. Se recurre a ella para tomar apuntes y utilizar aplicaciones que exigen un mayor rendimiento del que pueda ofrecer el smartphone.

En tercer lugar, la mayoría de las universidades del mundo ofrecen acceso gratuito a la tecnología wifi dentro del aula. En este sentido, se puede observar un movimiento creciente entre la mayoría de las universidades de todo el mundo, desde la primera década del siglo XXI, para proporcionar acceso universal a los distintos dispositivos móviles a todos sus estudiantes. Este movimiento ha sido impulsado por los gestores de esas instituciones, plenamente convencidos de que el uso de las nuevas TIC podría representar un cambio positivo en el enfoque de enseñanza-aprendizaje, bajo la premisa de que el acceso universal a los dispositivos móviles debería conducir a un aprendizaje mucho más efectivo (Demb et al., 2004). De hecho, esta idea se vio respaldada por la existencia de una creciente evidencia, tanto con base estadística como anecdótica, que parecía confirmar que el uso de las nuevas TIC conducía a mejores resultados para los estudiantes.

En cuarto lugar, los profesores han ido adaptando de manera paulatina sus metodologías y enfoques de enseñanza para acomodar el uso de las nuevas TIC en sus asignaturas. En parte, esto también ha sido una consecuencia de la implementación del conocido como 
«Proceso de Bolonia», donde las nuevas tecnologías y el aprendizaje a distancia se han convertido en aspectos clave en la mayoría de los programas académicos. Además, parece obvio que ha habido una necesidad de modificar las nuevas prácticas educativas porque las competencias y habilidades digitales están desempeñando un papel cada vez más relevante en la forma en que las personas aprenden. En este marco, las nuevas TIC se han convertido también en un activo clave para fomentar la innovación y la creatividad en los negocios y en la sociedad (Comisión Europea, 2008). De hecho, en las titulaciones universitarias la adquisición de diferentes tipos de competencias estrechamente vinculadas con el uso de las nuevas TIC se ha convertido en un aspecto esencial.

Con respecto al impacto del uso de los dispositivos móviles en el aula en contextos de educación superior, es importante tener en cuenta que hay poco consenso en la literatura sobre el impacto de su utilización en el rendimiento de los estudiantes. Por un lado, en un buen número de estudios realizados hasta el momento se han reconocido una serie de beneficios potenciales. Específicamente, se ha demostrado que los dispositivos móviles ayudan al aprendizaje a través de la utilización de enfoques más activos de enseñanza (véase Finn e Inman, 2004), proporcionando el ímpetu necesario para que los docentes procuren que sus materias sean más atractivas para los estudiantes, facilitando una mayor interacción entre docentes y estudiantes, y con ello la participación en clase, y aumentando así el compromiso, la satisfacción y el aprendizaje de los estudiantes (véanse, por ejemplo, Enríquez, 2010; Fried, 2008; Lindroth y Bergquist, 2010; Remón et al. , 2017; Wakefiled et al. , 2018; Weaver y Nilson, 2005; Willemse et al., 2019). Algunos estudios también revelan que estos dispositivos son beneficiosos para desarrollar diferentes tipos de habilidades de comunicación y pensamiento crítico de los estudiantes (véanse, por ejemplo, Bagdasarov et al., 2017; Ramamuruthy y Rao, 2015).

Otros estudios también han encontrado que, cuando los dispositivos móviles se usan con fines académicos -tomar notas y usar diferentes programas de software (véanse, por ejemplo, Driver, 2002; Kay y Lauricella, 2014), buscar información adicional o verificar determinados hechos/datos (véanse, por ejemplo, Aguirre et al., 2019; Berry y Westfall, 2015; Gay et al., 2001; Leyrer-Jackson y Wilson, 2018; Roberts y Rees, 2014), acceder a recursos suplementarios y a actividades basadas en la web (véanse, por ejemplo, Albó et al., 2019; Debevec et al., 2006), organizar y proporcionar acceso a materiales relacionados con la materia en cuestión (véanse, por ejemplo, Aguirre et al., 2019; Carter et al., 2017), o, incluso, ver diapositivas (véanse, por ejemplo, Aguirre et al., 2019; McVay et al., 2005; Roberts y Rees, 2014)-, pueden contribuir de manera significativa a aumentar el compromiso, la satisfacción y la motivación de los estudiantes (véanse, por ejemplo, Aguirre et al., 2019; Albó et al., 2019; Fried, 2008; Hyden, 2005; Samson, 2010; Weaver y Nilson, 2005). Algunos estudios también han 
confirmado que el uso de los dispositivos móviles puede marcar una diferencia sustancial en los hábitos de estudio de los estudiantes $\mathrm{y}$, en última instancia, en sus vidas académicas y sociales, ya que su uso puede ayudar a promover una mayor colaboración entre ellos, un intercambio de recursos e incluso una mejor organización personal (véanse, por ejemplo, Aguirre et al., 2019; Bagdasarov et al., 2017; Kay y Lauricella, 2014; Lauricella y Kay, 2010; Nicol y MacLeod, 2005; Ramamuruthy y Rao, 2015; Rambe y Bere, 2013).

Por contra, un gran número de investigaciones sugieren que los estudiantes que usan dispositivos móviles en clase exhiben más bajos niveles de satisfacción con su educación, obtienen peores experiencias de aprendizaje en comparación con los estudiantes que no los utilizan, tienen más probabilidades de realizar múltiples tareas en clase y, en consecuencia, están más distraídos (véanse, por ejemplo, McCabe y Meuter, 2011; Sage et al., 2020; Witecki y Nonnecke, 2015; Wurst et al., 2008; Zhang, 2015). Y todo ello sin tener en cuenta los problemas de adicción que su uso puede generar también, con las consiguientes consecuencias negativas ya no solo a nivel académico, sino también desde un punto de vista social. Algunos trabajos muestran que la mayoría de los estudiantes son conscientes de que el uso de dispositivos digitales en el aula les afecta en términos de falta de atención a las explicaciones del docente (véase, por ejemplo, McCoy, 2016). En este sentido, varios estudios, utilizando cuestionarios realizados a estudiantes y observaciones en el aula, han puesto de relieve que los dispositivos móviles se están usando con fines no académicos, como la mensajería instantánea y los juegos online (véanse, por ejemplo, Barak et al., 2006; Driver, 2002; Kay y Lauricella, 2014), el visionado de películas o de correos electrónicos (véanse, por ejemplo, Finn e Inman, 2004; Kay y Lauricella, 2014; Lauricella y Kay, 2010), la navegación por la web o incluso la realización de compras online (véanse, por ejemplo, Bugeja, 2007; Fried, 2008; Kay y Lauricella, 2014; McCreary, 2009). A veces se utiliza como justificación que la facilidad para el acceso al entretenimiento en línea durante las clases hace realmente difícil que lo que explican muchos docentes sea «más interesante que [lo que se puede ver en] YouTube» (Associated Press, 2010, p. 10), sobre todo cuando los estudiantes no están especialmente motivados por la asignatura.

Si bien los datos sobre cuánto tiempo los estudiantes están usando sus dispositivos móviles en clase pueden variar de manera significativa, el efecto de la distracción individual y social del uso de estos dispositivos para actividades no académicas resulta innegable (véanse, por ejemplo, Glass y Kang, 2019; Kim et al., 2019; Kraushaar y Novak, 2010; Sana et al., 2013). En esta línea, algunas investigaciones informan de que una proporción sustancial del tiempo de clase generalmente se pierde en actividades de índole no académica (véanse, 
por ejemplo, Rabl et al., 2019; Ragan et al., 2014). Es importante destacar que la distracción derivada de la realización de múltiples tareas en clase se relaciona también con reducciones significativas en el aprendizaje y las notas obtenidas (véanse, por ejemplo, Wammes et al., 2019; Wei et al., 2014). Los estudiantes que realizan múltiples tareas con los dispositivos móviles durante el tiempo de clase tienden a mostrar más problemas de comprensión del material o de las explicaciones dadas en clase, esto es, tienen peor memoria o capacidad cognitiva para el procesamiento de la información transmitida durante la clase por el personal docente (véanse, por ejemplo, Barak et al., 2006; Demirbilek y Talan, 2018; Jamet et al., 2020; Kraushaar y Novak, 2010; Sana et al., 2013).

\subsection{Estudios empíricos sobre la relación entre el uso de dispo- sitivos móviles en las aulas universitarias y el rendimiento académico de los estudiantes}

Ciertamente, como se ha señalado, mientras que algunos estudios han demostrado que la presencia de distintos dispositivos móviles en el aula reporta importantes beneficios, otros muchos también han documentado desventajas relevantes. Estos hallazgos contradictorios ponen de manifiesto la necesidad de identificar si estos dispositivos móviles realmente mejoran o empeoran el rendimiento académico de los estudiantes universitarios. En consecuencia, este es un tema clave que debe abordarse y aclararse, dado que uno de los objetivos más importantes de los estudiantes es aprobar las diversas materias. Obviamente, este objetivo también es compartido por los docentes. Es por ello que nuestro interés en este trabajo se centra también en revisar una lista representativa de estudios empíricos que hayan analizado la relación entre el uso de los diferentes tipos de dispositivos móviles en el aula y el rendimiento académico de los estudiantes universitarios de ambos sexos.

Con objeto de poner de manifiesto la evolución de la producción científica en esta área desde una perspectiva amplia y demostrar su relevancia actual y potencial recorrido futuro, los referidos estudios han sido identificados y recuperados de Web of Science (WOS). Para ello, en mayo de 2020 se realizó una búsqueda sistemática de literatura en dicha base de datos para el periodo 2000-2020, utilizando como criterios de búsqueda en «topics» los siguientes términos: ([«mobil ${ }^{\star}$ device* ${ }^{\star}$ or «laptop*» or «comput ${ }^{\star} »$ or «tablet ${ }^{\star} »$ or «smartphon*»]

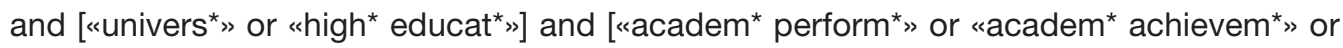
"academ* outcom*»]). La búsqueda dentro de la colección principal de WOS se limitó a Science Citation Index Expanded (SCI_EXPANDED), Social Sciences Citation Index (SSCI), Art \& Humanities Citation Index (A\&HCl) y Emerging Sources Citation Index (ESCl). Estos criterios de búsqueda proporcionaron 421 artículos (409 artículos publicados; 1 repositorio de datos y 11 artículos publicados con asignación de número de revista pendiente). La figura 1 muestra la producción científica anual de artículos con los criterios de búsqueda señalados en WOS. Se observa una importante tendencia creciente de publicación en esta área en los últimos años. 
Figura 1. Producción científica anual sobre el uso de los dispositivos móviles en contextos de educación superior en WOS (2000-2020)

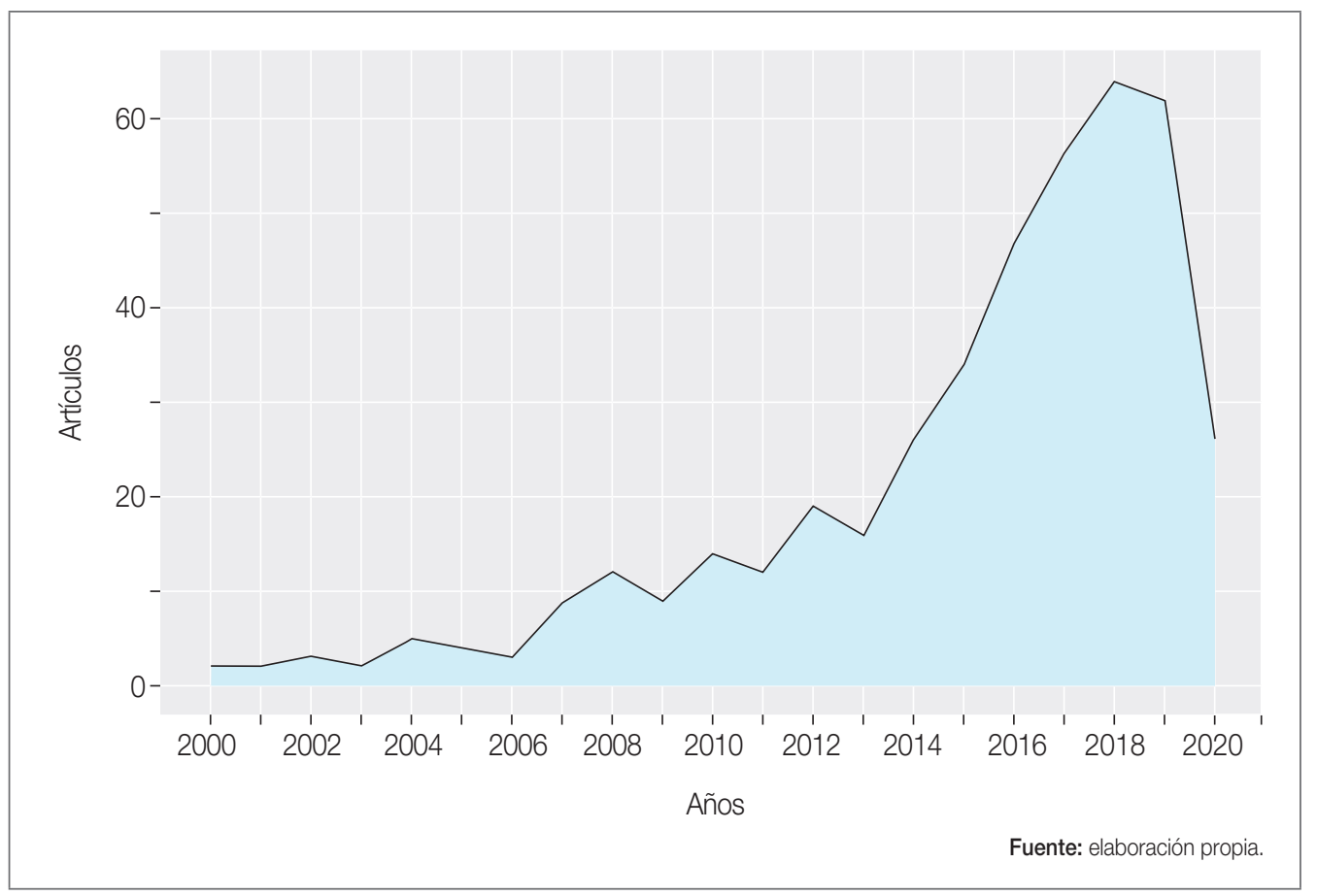

Tras identificar los potenciales trabajos existentes sobre el tema en WOS, se realizó la lectura del título y abstract de cada uno de ellos para evaluar si el artículo encajaba en el estudio del tópico en cuestión. Este proceso redujo de manera significativa el número de artículos a considerar en la revisión. Por último, se realizó un proceso de búsqueda manual y de seguimiento de citas. En este caso, se optó por incluir algún trabajo adicional que figura en el primer cuartil del SCimago Journal Rank y que, en la mayoría de los casos, cuenta con un número de citas elevado (según Google Scholar) y, además, se cita en los artículos de WOS identificados en un primer momento. De este modo, el número total de artículos revisados asciende finalmente a 67.

El cuadro del anexo resume los principales hallazgos de los 67 estudios finalmente considerados en nuestra revisión. Todos ellos han sido publicados en revistas de impacto y de reconocido prestigio dentro del ámbito académico. En dicho cuadro se muestra información sobre los autores, el año del estudio (ordenados cronológicamente), los participantes y el contexto universitario en el que se realiza, los principales resultados/hallazgos del estudio en cuestión, así como el número de citas (en términos absolutos y relativos) recibidas en Google Scholar. Puede comprobarse que la evolución anual del número de artículos considerados en la revisión entre 2000-2020 muestra una tendencia similar a la producción científica anual que se ilustra en la figura 1. 
El estudio de Maki et al. (2000) se encuentra entre los primeros en realizar un análisis estadístico sobre la relación considerada. Estos investigadores encontraron que los estudiantes que empleaban ordenadores para el seguimiento de la asignatura obtenían un mejor conocimiento de la asignatura y, también, una mejor nota en los exámenes. Este trabajo, por tanto, proporcionaba una visión bastante optimista sobre el uso de los ordenadores en el aula. El cuadro del anexo sintetiza la información del resto de investigaciones cuantitativas identificadas sobre el tema. A la vista de la información que figura en este cuadro pueden extraerse una serie de conclusiones:

- La mayoría de investigaciones se han llevado a cabo desde la primera década del siglo XXI, que es precisamente el momento a partir del que se empieza a generalizar el uso de los distintos dispositivos móviles en las aulas, en particular, los ordenadores portátiles y las tablets. No obstante, la inmensa mayoría (más del $80 \%$ de las publicaciones) se han realizado entre 2010-2020 y más de la mitad entre 2015-2020. Estas cifras no hacen sino constatar la creciente importancia que ha ido adquiriendo el tópico. De esto se puede deducir también que es aún un tema de investigación bastante atractivo por el elevado interés que sigue despertando.

- Más de la mitad de los estudios se han realizado en universidades de EE. UU. (casi el $55 \%$ de los estudios analizados) o de influencia anglosajona (en torno al $75 \%$ del total).

- Alrededor del $40 \%$ de los estudios analizados ponen el foco de manera exclusiva en el uso de los ordenadores -ya sean ordenadores portátiles o tablets-, el $20 \%$ en el uso de los smartphones y el resto en el uso de cualquier dispositivo móvil sin distinción. De hecho, mientras los estudios iniciales se centraban sobre todo en el uso de los ordenadores, los estudios realizados a partir de 2010 empiezan a poner también el foco en el uso de los smartphones.

- En términos de impacto de estos dispositivos sobre el rendimiento académico de los estudiantes, a primera vista los resultados son bastante reveladores al respecto: el uso de los dispositivos móviles lleva consigo la obtención de peores notas. Esto es lo que han encontrado casi un $60 \%$ de los estudios analizados. Por el contrario, alrededor del $25 \%$ de los estudios han detectado que su uso en clase repercute de manera positiva sobre la nota final, mientras que el resto encuentra que no existen diferencias significativas o resultados ambiguos en función de la facultad, país, asignatura o año cursado.

Por tanto, dada la disparidad de hallazgos existentes, pensamos que es importante realizar más investigaciones que ahonden en el estudio de la relación entre el uso de dispositivos móviles en el aula y el rendimiento académico de los estudian-

La inmensa mayoría de las publicaciones (más del $80 \%$ ) se han realizado entre $2010-2020$ y más de la mitad entre 2015-2020 tes universitarios. Con ello daríamos respuesta a la 
primera de las preguntas planteadas en nuestro trabajo. No obstante, a diferencia de la mayoría de los estudios previos, nuestro trabajo también se hace eco de la necesidad de controlar los siguientes aspectos:

- Materia que imparte el docente.

- Ciertas características de los docentes.

- Año de estudio en la universidad.

En suma, en este trabajo, estamos especialmente interesados en explorar si estos aspectos pueden tener, al menos a priori, algún tipo de influencia en la relación considerada, aunque inicialmente no asumimos que dicha influencia sea en uno u otro sentido. De este modo, se daría respuesta también a la segunda pregunta planteada.

\section{Metodología}

La muestra utilizada en este trabajo consistió en 176 estudiantes de ambos sexos de educación superior de una de las universidades más grandes de España (más de 46.000 estudiantes). Todos los estudiantes estaban matriculados en un grado relacionado con la administración de empresas, pertenecían a clases distintas, tenían tres docentes diferentes y asistían habitualmente a clase. Con respecto al año universitario, 46 estudiantes estaban en su primer año, cursando una asignatura introductoria sobre administración de empresas (asignatura A), mientras que 130 estudiantes estaban en su tercer año, cursando una asignatura sobre dirección financiera. Las tres asignaturas se impartieron durante el primer semestre del curso 2019-2020. Los estudiantes matriculados en la asignatura A estaban en la misma clase, mientras que los estudiantes de dirección financiera estaban distribuidos en dos clases que contaban con 72 y 48 estudiantes y fueron impartidas por dos docentes diferentes (asignatura B y asignatura $\mathrm{C}$, respectivamente).

Los tres docentes (en concreto, dos profesores y una profesora) habían participado en numerosos cursos de formación relacionados con el uso de las nuevas TIC en la educación. Dichos cursos fueron impartidos por profesionales de la misma universidad, que contaban con una amplia experiencia en el uso de estas tecnologías. Los tres docentes también tenían un doctorado en el campo de la administración de empresas y contaban con cerca de 20 años de experiencia docente. Los tres docentes tenían estilos de enseñanza bien diferenciados. Específicamente, mientras el docente de la asignatura $A$ utilizó principalmente las TIC en sus clases, los docentes de las asignaturas B y C tendían a utilizar en gran medida las herramientas de enseñanza clásicas, como la pizarra, aunque permitían en sus clases el uso de distintos dispositivos móviles. 
En todo caso, conviene reseñar que el uso de smartphones estaba prohibido en las tres clases. Además, los estudiantes que usaban ordenadores portátiles o tablets durante la clase generalmente estaban sentados juntos (es decir, en la misma zona del aula). Del mismo modo, los estudiantes que nunca usaron tales dispositivos también solían sentarse juntos. Por tanto, de esta forma también se controlaban los posibles efectos de distracción social relacionados con el uso de ordenadores portátiles/tablets, sobre todo en el caso de aquellos estudiantes que no los utilizaban.

Más allá del uso de ordenadores portátiles y tablets, las estructuras de las clases y las formas de evaluación fueron bastante similares en las tres asignaturas. Por otro lado, todos los estudiantes tenían acceso al material de la materia, que podían descargar o imprimir antes de cada clase con antelación suficiente para, de este modo, facilitarles en la medida de lo posible el proceso de seguimiento y aprendizaje. Sorprendentemente, en aquellos casos en que las clases requerían escribir fórmulas, los estudiantes que usaban ordenadores portátiles/ tablets encontraban muchas más dificultades para el seguimiento normal de la clase, ya que a menudo requerían de la ayuda de los docentes una vez concluidas las clases para la aclaración de distintas cuestiones relacionadas con el contenido impartido.

Con respecto al proceso de evaluación, las preguntas de los exámenes fueron de similar dificultad en todas las materias y consistieron en un porcentaje parecido de preguntas tipo test de opción múltiple y de respuesta corta. En línea con estudios previos (véanse, por ejemplo, Carter et al., 2017; Hutcheon et al., 2019; Kolar et al., 2002; Kraushaar y Novak, 2010; Patterson y Patterson, 2017), se utilizó la calificación final obtenida en cada asignatura como un indicador de rendimiento académico.

La mayoría de los trabajos del cuadro del anexo son de naturaleza experimental y hacen uso de cuestionarios, de modo que la información relativa tanto al uso de los dispositivos móviles como al rendimiento académico es proporcionada por los propios estudiantes. Nuestro estudio se basa en el seguimiento directo por parte del docente, a lo largo de un semestre, de los estudiantes que llevaban o no a las clases portátiles o tablets y la información de las calificaciones se obtuvo de las actas de cada asignatura. El tratamiento de la información se realizó garantizando el anonimato de los estudiantes en todo momento.

Desde el punto de vista del análisis empírico, para dar respuesta a las dos preguntas planteadas, se hizo uso de «t-test para muestras independientes», dado que los datos recopilados cumplían con los supuestos estadísticos requeridos para su aplicación; a saber, las medias de las dos series de datos (grupo de estudiantes que usaban habitualmente ordenadores portátiles/tablets durante la clase y grupo de estudiantes que nunca los empleaban) que se comparaban seguían distribuciones normales y tenían la misma varianza. Los test de diferencias de medias en sus distintas versiones han sido también la herramienta estadística empleada por la mayoría de trabajos que figuran en el cuadro del anexo. 


\section{Resultados}

El cuadro 1 muestra los principales resultados de los diferentes «t-test» realizados. Específicamente, llevamos a cabo cuatro «t-test» por separado. En el primer caso, consideramos a todos los estudiantes sin distinguir la asignatura específica que estaban cursando $(n=176)$, mientras que, en los tres casos restantes, consideramos solo a los estudiantes que estaban cursando cada materia (es decir, las asignaturas $A, B$ y $C$, respectivamente).

Cuadro 1. Rendimiento académico de estudiantes usando dispositivos móviles en clase versus estudiantes que no los utilizan nunca

\begin{tabular}{|c|c|c|c|c|c|}
\hline \multirow[t]{2}{*}{ Asignatura } & $\begin{array}{l}\text { Usan ordenador } \\
\text { portátil/Tablet }\end{array}$ & $\begin{array}{l}\text { No usan ordena- } \\
\text { dor portátil/Tablet }\end{array}$ & \multirow[t]{2}{*}{$t$} & \multirow[t]{2}{*}{$g l$} & \multirow[t]{2}{*}{$p$-valor } \\
\hline & Nota media & Nota media & & & \\
\hline $\begin{array}{l}\text { Todas las asignaturas } \\
(n=176)\end{array}$ & 4,005 & 4,091 & 0,247 & 216,45 & 0,805 \\
\hline Asignatura A $(n=46)$ & 6,100 & 4,996 & 1,853 & 40,45 & 0,079 \\
\hline Asignatura B $(n=72)$ & 2,868 & 3,904 & 1,873 & 58,96 & 0,066 \\
\hline Asignatura C $(n=58)$ & 4,005 & 4,091 & 0,608 & 42,98 & 0,546 \\
\hline
\end{tabular}

Nota: $t=$ Valor del estadístico $t$ en el $t$-test; $g l=$ Grados de libertad; $p$-valor = Significación estadística.

Fuente: elaboración propia.

El cuadro 1 muestra que no existían diferencias estadísticamente significativas en la nota final media entre el grupo de estudiantes que usaban y no usaban dispositivos móviles durante las clases cuando se consideró toda la muestra de estudiantes. Sin embargo, cuando se desglosaron los estudiantes por cada asignatura cursada de manera individual, se obtuvieron los siguientes hallazgos. Para los estudiantes que estaban cursando la asignatura $A$, encontramos que existen diferencias estadísticamente significativas (aunque débilmente, $p<0,10$ ) en la nota final media entre ambos grupos de estudiantes. En concreto, se observa que el grupo de estudiantes que usa tales dispositivos durante la clase logra notas finales medias más altas que el grupo de estudiantes que no hacen uso de ellos. Para los estudiantes de la asignatura B, encontramos justo lo contrario: el grupo de estudiantes que usaban ordenadores portátiles/tablets obtenían notas finales medias más bajas. Finalmente, para los estudiantes de la asignatura $\mathrm{C}$, no se encontraron diferencias estadísticamente significativas en las notas finales medias entre ambos grupos. 
Por tanto, a partir de nuestro estudio, la respuesta a la primera pregunta (¿Existen diferencias significativas en el rendimiento académico entre el grupo de estudiantes universitarios que utiliza con frecuencia ordenadores portátiles o tablets durante la clase y el grupo de estudiantes que nunca los usa en clase?) no es inequívoca. Al contrario, nuestros resultados parecen sugerir que la nota lograda por los estudiantes puede depender de algunos otros aspectos o factores. En este sentido, observamos que las diferencias significativas identificadas podrían depender a priori de los siguientes factores: la asignatura concreta que se está enseñando por el docente, el año de estudio, ciertas características/intereses de los docentes e incluso el tamaño de la clase. Respecto a este último aspecto, es importante señalar que los resultados de los estudiantes que usan dispositivos móviles en clase respecto a los que no los utilizan son peores cuanto mayor es el tamaño de la clase.

En consecuencia, nuestra respuesta a la segunda pregunta (¿Las diferencias significativas en el rendimiento académico se producen independientemente de la materia que cursan los estudiantes, de los docentes que imparten la clase y/o del año de estudio en que se encuentran?) es que dichos aspectos o factores pueden desempeñar un papel importante en la explicación de las diferencias detectadas.

\section{Conclusiones y algunas propuestas para la reflexión}

La cuestión de si hay que prohibir el uso de dispositivos móviles en las aulas de las universidades o si debe permitirse su uso en vista del impacto que pueden tener sobre el rendimiento académico de los estudiantes ha suscitado en los últimos años, y sigue haciéndolo, un acalorado debate en el campo educativo, en general, y en la educación universitaria, en particular. Si tomamos como referencia las investigaciones previas realizadas sobre este tema en las últimas dos décadas (véase el cuadro del anexo), podemos señalar que todavía no existe una respuesta completamente satisfactoria a dicha cuestión.

Específicamente, a la luz de la revisión de la literatura realizada aquí, parece obvio que este ámbito de investigación está aún lejos del consenso, si bien, de los trabajos analizados hasta la fecha, parece desprenderse (de manera mayoritaria) una visión bastante pesimista acerca de sus efectos reales sobre las calificaciones finales de los estudiantes, lo que vendría a justificar, en cierta medida, la posición de aquellos que abogan por su prohibición. No obstante, es razonable concluir que este campo de investigación, aunque parece estar ya bastante consolidado, aún no ha alcanzado el grado de madurez deseado (definido por la existencia de un alto grado de consenso empírico). Por tanto, parece más que justificada la realización de nuevas investigaciones para ver en qué medida los resultados pueden ser o no más ampliamente generalizables.

El objetivo principal de este estudio ha sido precisamente proporcionar nueva evidencia que sea útil a la hora tomar una decisión más informada sobre la conveniencia de prohibir o 
permitir el uso de estos dispositivos móviles en las aulas universitarias a la vista de su impacto sobre el rendimiento académico de los estudiantes. En este sentido, nos hemos centrado en identificar si existen diferencias estadísticamente significativas en la calificación final entre el grupo de estudiantes que habitualmente usan estos dispositivos durante la clase y el grupo que nunca los utiliza. Es importante destacar que hemos prestado especial atención a la necesidad de considerar algunos aspectos que la mayoría de los estudios anteriores han pasado por alto.

En general, nuestros resultados, así como los de estudios previos, ofrecen soporte empírico para argumentar que la utilización de dispositivos móviles en las clases es un tema complejo que debe evaluarse cuidadosamente. En cierto modo, nuestros resultados sugieren que no hay una respuesta a la pregunta anterior que sea universalmente válida. Esto es así porque en el caso de una asignatura (A), hemos obtenido evidencia a favor del uso de ordenadores portátiles/tablets durante la clase, en la medida en que pueden ayudar a mejorar significativamente la nota final de los estudiantes. Este hallazgo está en línea con aquellos estudios que también proporcionan una visión positiva/optimista sobre el uso de estos dispositivos en clase, en términos de su efecto sobre el rendimiento académico (véase anexo). De alguna manera, estos resultados sugieren que en esta asignatura en concreto los beneficios potenciales relacionados con el uso de dispositivos móviles durante la clase superan sus potenciales desventajas. Es importante tener en cuenta que esta asignatura se imparte en el primer año universitario y, en comparación con las otras asignaturas, el número de estudiantes también es bastante menor. Resulta obvio que es más fácil para el docente controlar comportamientos inapropiados con tales dispositivos si el número de estudiantes en clase es pequeño.

Sin embargo, en otra asignatura (B) hemos encontrado que la nota final media de los estudiantes que usan dispositivos móviles durante la clase es significativamente menor en comparación con los estudiantes que nunca los utilizan. Este hallazgo también es consistente con estudios que tienden a sugerir una visión más pesimista sobre la utilidad real de estos dispositivos en el aula (véase anexo). Es importante tener en cuenta que esta fue la asignatura con el mayor número de estudiantes de las tres examinadas. Además, en este caso, el docente escribía frecuentemente en la pizarra para explicar muchas cuestiones clave del contenido de la asignatura. Finalmente, en otra asignatura (C) no encontramos diferencias estadísticamente significativas en la nota final media entre los grupos de estudiantes que usan o no dispositivos móviles durante la clase. Este docente también escribía en la pizarra para explicar el contenido de la asignatura. El resultado está también en consonancia con los obtenidos en otras investigaciones (véase anexo). Estos hallazgos sugieren que, en cierta medida, en ambas asignaturas (pero, especialmente, en la B) las posibles desventajas relacionadas con el uso de dispositivos móviles durante la clase superan con creces sus potenciales ventajas.

En el estudio de Martínez et al. (2019) se pone de manifiesto que la insuficiente formación tecnológica de los docentes universitarios (españoles) desempeña un papel clave a la hora de adoptar una postura contraria a la innovación con dispositivos móviles. Esta cuestión, que podría esgrimirse para justificar algunos de los resultados obtenidos, no sería aplicable a nuestro estudio empírico, puesto que los tres docentes han participado asiduamente 
en numerosos cursos de formación relacionados con el uso de las nuevas TIC en el ámbito universitario y, por tanto, están ampliamente familiarizados con su utilización en las clases. En cualquier caso (e incluso siendo conscientes de las posibles limitaciones asociadas al tamaño muestral), estamos convencidos de que nuestros hallazgos pueden deberse también al contexto de la clase, así como a otros factores potenciales que no se han controlado en este estudio, más que a un mero efecto estadístico.

Si bien el análisis desarrollado en el presente trabajo nos ha permitido controlar de algún modo los resultados por aspectos como son la materia impartida, las características del docente y el año de impartición de la asignatura, empleando así un nivel de desagregación de datos mayor que el utilizado en la gran mayoría de investigaciones sobre el tema -con algunas excepciones que hacen uso de una desagregación similar, como son Gaudreau et al. (2014), Grace-Martin y Gay (2001), Le Roux y Parry (2017), Kolar et al. (2002) y Pemberton et al. (2006), entre otros-, es importante señalar que existen otros factores no contemplados en el análisis que pueden influir en los resultados obtenidos. Así, Sulaiman y Mohezar (2006) explican cómo el rendimiento académico de los estudiantes en cursos anteriores o en estudios cursados previamente es un indicador de primer orden del rendimiento académico posterior. Por otro lado, Nonis y Hudson (2010) estudian el papel del tiempo de estudio y de los hábitos de estudio sobre el rendimiento académico de los estudiantes, obteniendo resultados mixtos dependiendo de los hábitos de estudio concretos analizados. Es por ello que, en futuras investigaciones sobre el impacto del uso de dispositivos móviles dentro del aula en el rendimiento de los estudiantes, la introducción de variables de control por rendimiento académico pasado, tiempo de estudio y hábitos de estudio permitirá aislar en mayor medida la interrelación existente entre ambas variables, así como medir de una manera más nítida el efecto de los dispositivos móviles en el aula sobre las calificaciones de los estudiantes.

Adicionalmente, es importante señalar que, para las tres asignaturas analizadas en el presente estudio, los estudiantes han utilizado los ordenadores portátiles y otros dispositivos móviles fundamentalmente para la toma de apuntes y la resolución de ejercicios. Sin embargo, el constante desarrollo de aplicaciones informáticas concebidas como herramientas de apoyo a la actividad del profesorado está ampliando de manera muy significativa el abanico de posibilidades al alcance de docentes y estudiantes en el desarrollo de las clases (Hanus y Fox, 2015; Çakıroğlu et al., 2017; Sánchez et al., 2020), lo que implica que investigaciones futuras sobre el tema deberán abordar en profundidad el efecto que los distintos usos de la tecnología en el aula pueden implicar sobre el rendimiento de los estudiantes.

A partir de todo lo expuesto anteriormente, estamos en disposición de sugerir una serie de propuestas que lanzamos para la reflexión y el debate:

- Prohibir el uso de dispositivos móviles en clase no debería considerarse, salvo situaciones muy puntuales, la mejor decisión. Es importante considerar que las nuevas TIC en el campo educativo han llegado para quedarse. Así pues, planteamos que la pregunta de si el uso de dispositivos móviles debe permitirse o prohibirse 
en el aula debe reemplazarse por la siguiente: ¿cómo pueden los docentes conseguir sacar el mejor partido posible del uso de los dispositivos móviles en sus clases? Aquí, en consonancia con otros investigadores (por ejemplo, Fried, 2008), sugerimos que es crucial encontrar formas alternativas de promover el uso más adecuado de estos dispositivos, mientras se reducen simultáneamente los posibles impactos negativos de un uso inapropiado. Obviamente, esto puede depender de la asignatura que se enseña, de los intereses del docente con dicha asignatura u otros fac-

¿Cómo pueden los docentes conseguir sacar el mejor partido posible del uso de los dispositivos móviles en sus clases?
Parece claro que cuanto menor sea el tamaño de la clase más posibilidades existirán de que el docente controle un posible uso inapropiado de los diferentes dispositivos móviles tores relacionados con el contexto de la clase, como puede ser, por ejemplo, su tamaño. En este último caso, parece claro que cuanto menor sea el tamaño de la clase más posibilidades existirán de que el docente controle un posible uso inapropiado de los diferentes dispositivos. Ciertamente, los docentes deben esforzarse por informar continuamente a los estudiantes sobre las posibles consecuencias negativas de un uso inapropiado de dichos dispositivos en sus resultados académicos. Pero los responsables de la gestión universitaria deberían hacer igualmente un esfuerzo no solo por facilitar los medios tecnológicos más adecuados en las aulas y con ello la labor docente, sino también por garantizar que los docentes puedan impartir sus clases con un número manejable de estudiantes.

- Tal vez es un buen momento para que los responsables en materia educativa se replanteen la necesidad de incluir en los programas de la mayoría de los títulos universitarios, a nivel de grado y equivalente, la impartición, por parte de expertos, durante el primer año académico, de una asignatura relacionada con el uso eficaz de las nuevas TIC. De hecho, muchos de los estudios analizados en este trabajo se centran en estudiantes de primer año en la universidad. Dicha asignatura debería tener un carácter obligatorio y proporcionar a los estudiantes toda la información relevante tanto sobre la utilidad y los principales beneficios potenciales asociados al uso de estas herramientas en sus diferentes variantes como sobre los posibles efectos negativos que pueden llevar aparejados debido a un uso inapropiado, que en numerosas ocasiones acaba desembocando en graves problemas de adicción. De este modo, los estudiantes tendrían también una perspectiva más global sobre el futuro uso de tales tecnologías. Esto serviría a los estudiantes para disponer de mayor información y poder tomar así una mayor y mejor conciencia del uso de tales tecnologías. De la misma manera, en la medida de lo posible, se le debería exigir al profesorado contar con la formación precisa para manejar eficazmente las nuevas tecnologías en sus respectivas clases. 
- Si las nuevas tecnologías son realmente «prometidas» en las diferentes titulaciones y/o asignaturas, pero luego no se proporcionan durante el proceso formativo de manera adecuada, es indudable que los estudiantes podrían tener argumentos para estar menos motivados y, en última instancia, menos comprometidos con sus futuras profesiones. En este sentido, debemos ser conscientes de que, actualmente, la mayoría de las profesiones laborales requieren como requisito indispensable ser competentes o expertos en el uso de determinadas herramientas tecnológicas. En consecuencia, en la medida de lo posible, la enseñanza en las aulas debería asemejarse a aquellas situaciones a las que deberán hacer frente los estudiantes en la práctica, es decir, en el ejercicio posterior de sus profesiones, ayudándoles a creer que su aprendizaje es importante y, también, lo más realista posible. En este sentido, también debemos ser conscientes de que, actualmente, estos dispositivos permiten realizar múltiples ejercicios de simulación para vislumbrar cómo pueden desarrollarse las cosas en el mundo real. En todo caso, a los estudiantes también se les debería hacer comprender que hay algunos conceptos para los que el uso de las nuevas tecnologías puede resultar una herramienta especialmente propicia, mientras que, para otros, tal vez, no tanto, requiriendo la combinación de métodos de corte más tradicional.

- En línea con lo expuesto en último lugar, debería tenerse también claro que ninguna tecnología constituye una panacea por sí misma. Por tanto, la comunidad educativa ha de tener presente que muchos de los problemas tradicionales asociados con la educación de los estudiantes seguirán estando presentes, sin importar cuán sofisticados puedan ser los dispositivos electrónicos que se utilicen. Lo que en cierto modo parece también obvio es que no siempre se puede dar una respuesta satisfactoria a «problemas nuevos» con «soluciones viejas». De ahí que se requiera un esfuerzo adicional, por parte de todos los agentes implicados, de adaptación a los nuevos tiempos mediante la adopción de algunas soluciones innovadoras vinculadas al uso eficaz de las nuevas tecnologías, lo cual debería redundar en beneficio de la provisión de una enseñanza de mayor calidad.

- La evidencia reciente pone de relieve que el uso de las nuevas TIC adquiere especial relevancia en un entorno de crisis como el que estamos experimentando actualmente: la crisis relacionada con la COVID-19. Las herramientas tecnológicas que posibilitan la colaboración y el teletrabajo se han convertido en esenciales en una situación como esta, y es muy probable que, para la mayoría de las profesiones, se conviertan en una tendencia imparable en el futuro cercano, tal y como indican la mayoría de las previsiones disponibles. Por consiguiente, es indudable que las universidades también son un lugar ideal para comenzar a preparar a los estudiantes con las herramientas tecnológicas que utilizarán en sus futuras profesiones y, claramente, una tarea importante de los docentes es ayudarles a usar estas herramientas de manera apropiada. 
Confiamos en que las propuestas aquí formuladas puedan contribuir al debate abierto en torno al uso de los dispositivos electrónicos en las aulas universitarias. Con ello se trataría de ayudar a articular algunos instrumentos que permitan optimizar el uso de tales dispositivos, en aras a la consecución de unos mejores resultados académicos por parte de los estudiantes, de los que, en última instancia, también se beneficiaría la socie-

Es indudable que las universidades también son un lugar ideal para comenzar a preparar a los estudiantes con las herramientas tecnológicas que utilizarán en sus futuras profesiones dad en su conjunto. Tales propuestas pueden ser tomadas en consideración por parte de todos aquellos agentes con algún tipo de responsabilidad en la toma de decisiones dentro del ámbito educativo, en general, y universitario, en particular, y, sobre todo, por aquellos que están en contacto más estrecho con los estudiantes, como es el caso del profesorado.

\section{Referencias bibliográficas}

Aguilar-Roca, N. M., Williams, A. E. y O'Dowd, D. K. (2012). The impact of laptop-free zones on student performance and attitudes in large lectures. Computers \& Education, 59(4), 1.300-1.308.

Aguirre, J., Chaves, S. y Burban, K. (2019). Adoption, adaptation, and use of mobiles by university students: a Central American example. Journal of Applied Research in Higher Education, 11(3), 493-505.

Albó, L., Hernández-Leo, D. y Moreno Oliver, V. (2019). Smartphones or laptops in the collaborative classroom? A study of video-based learning in higher education. Behaviour \& Information Technology, 38(6), 637-649.

Arain, A. A., Hussain, Z., Rizvi, W. H. y Vighio, M. S. (2018). An analysis of the influence of a mobile learning application on the learning outcomes of higher education students. Universal Access in the Information Society, 17(2), 325-334.

Artz, B., Johnson, M., Robson, D. y Taengnoi, S. (2020). Taking notes in the digital age: evi- dence from classroom random control trials. The Journal of Economic Education, 51(2), 103-115.

Associated Press. (2010). At universities, is better learning a click away? Education Week, 29.

Baert, S., Vujić, S., Amez, S., Claeskens, M., Daman, T., Maeckelberghe, A., Omey, E. y Marez, L. de. (2019). Smartphone use and academic performance: correlation or causal relationship? Kyklos, 73(1), 22-46. https:// doi.org/10.1111/kykl.12214

Bagdasarov, Z., Luo, Y. y Wu, W. (2017). The influence of tablet-based technology on the development of communication and critical thinking skills: an interdisciplinary study. Journal of Research on Technology in Education, 49(1-2), 55-72.

Barak, M., Lipson, A. y Lerman, S. (2006). Wireless laptops as means for promoting active learning in large lecture halls. Journal of Research on Technology in Education, 38(3), 245-263. 
Bellur, S., Nowak, K. L. y Hull, K. S. (2015). Make it our time: in class multitaskers have lower academic performance. Computers in Human Behavior, 53, 63-70.

Berry, M. J. y Westfall, A. (2015). Dial D for distraction: the making and breaking of cell phone policies in the college classroom. College Teaching, 63(2), 62-71.

Brooks, S. (2015). Does personal social media usage affect efficiency and well-being? Computers in Human Behavior, 46, 26-37.

Brown, D. G. y Petitto, K. R. (2003). The status of ubiquitous computing. Educause Review, 38(3), 24-33.

Brown, D. G., Burg, J. J. y Dominick, J. L. (1998). A strategic plan for ubiquitous laptop computing. Communications of the ACM, 41(1), 26-35.

Bugeja, M. J. (2007). Distractions in the wireless classroom. Chronicle of Higher Education, 53(21), C1-C4.

Çakıroğlu, Ü, Başıüyükb, B., Gülerc, M., Atabaya, M. y Memiş, B. Y. (2017). Gamifying an ICT course: influences on engagement and academic performance. Computers in Human Behavior, 69, 98-107.

Carle, A. C., Jaffee, D. y Miller, D. (2009). Engaging college science students and changing academic achievement with technology: a quasi-experimental preliminary investigation. Computers \& Education, 52(2), 376-380.

Carter, S. P., Greenberg, K. y Walker, M. S. (2017). The impact of computer usage on academic performance: evidence from a randomized trial at the United States Military Academy. Economics of Education Review, 56, 118-132.

Clayson, D. E. y Haley, D. A. (2013). An introduction to multitasking and texting: prevalence and impact on grades and GPA in marketing classes. Journal of Marketing Education, 35(1), 26-40.
Comisión Europea. (2008). The use of ICT to Support Innovation and Lifelong Learning for All-A Report on Progress. Commission Staff Working Document.

Crook, C. y Barrowcliff, D. (2001). Ubiquitous computing on campus: patterns of engagement by university students. International Journal of Human-Computer Interaction, 13(2), 245-256.

Debevec, K., Shih, M. Y. y Kashyap, V. (2006). Learning strategies and performance in a technology integrated classroom. Journal of Research on Technology in Education, 38(3), 293-307.

Demb, A., Erickson, D. y Hawkins-Wilding, S. (2004). The laptop alternative: student reactions and strategic implications. Computers \& Education, 43(4), 383-401.

Demirbilek, M. y Talan, T. (2018). The effect of social media multitasking on classroom performance. Active Learning in Higher Education, 19(2), 117-129.

Downs, E., Tran, A., McMenemy, R. y Abegaze, N. (2015). Exam performance and attitudes toward multitasking in six, multimedia-multitasking classroom environments. Computers \& Education, 86, 250-259.

Driver, M. (2002). Exploring student perceptions of group interaction and class satisfaction in the web-enhanced classroom. The Internet and Higher Education, 5(1), 35-45.

Dunn, T. J. y Kennedy, M. (2019). Technology enhanced learning in higher education; motivations, engagement and academic achievement. Computers \& Education, 137, 104-113.

Elliott-Dorans, L. R. (2018). To ban or not to ban? The effect of permissive versus restrictive laptop policies on student outcomes and teaching evaluations. Computers \& Education, 126, 183-200.

Ellis, Y., Daniels, B. y Jauregui, A. (2010). The effect of multitasking on the grade performance 
of business students. Research in Higher Education Journal, 8(1), 1-10.

Enríquez, A. G. (2010). Enhancing student performance using tablet computers. College Teaching, 58(3), 77-84.

Felisoni, D. D. y Godoi, A. S. (2018). Cell phone usage and academic performance: an experiment. Computers \& Education, 117, 175-187.

Finn, S. e Inman, J. G. (2004). Digital unity and digital divide: surveying alumni to study effects of a campus laptop initiative. Journal of Research on Technology in Education, 36(3), 297-317.

Fox, A. B., Rosen, J. y Crawford, M. (2009). Distractions, distractions: does instant messaging affect college students' performance on a concurrent reading comprehension task? Cyberpsychology \& Behavior, 12(1), 51-53.

Fried, C. B. (2008). In-class laptop use and its effects on student learning. Computers \& Education, 50(3), 906-914.

Froese, A. D., Carpenter, C. N., Inman, D. A., Schooley, J. R., Barnes, R. B., Brecht, P. W. y Chacon, J. D. (2012). Effects of classroom cell phone use on expected and actual learning. College Student Journal, 46(2), 323-332.

Gaudreau, P., Miranda, D. y Gareau, A. (2014). Canadian university students in wireless classrooms: what do they do on their laptops and does it really matter? Computers \& Education, 70, 245-255.

Gay, G., Stefanone, M., Grace-Martin, M. y Hembrooke, H. (2001). The effects of wireless computing in collaborative learning environments. International Journal of Human-Computer Interaction, 13(2), 257-276.

Giunchiglia, F., Zeni, M., Gobbi, E., Bignotti, E. y Bison, I. (2018). Mobile social media usage and academic performance. Computers in Human Behavior, 82, 177-185.
Glass, A. L. y Kang, M. (2019). Dividing attention in the classroom reduces exam performance. Educational Psychology, 39(3), 395-408.

Grace-Martin, M. y Gay, G. (2001). Web browsing, mobile computing and academic performance. Journal of Educational Technology \& Society, 4(3), 95-107.

Green, A. (2016). Significant returns in engagement and performance with a free teaching app. The Journal of Economic Education, 47(1), 1-10.

Hanus, M. D. y Fox, J. (2015). Assessing the effects of gamification in the classroom: a longitudinal study on intrinsic motivation, social comparison, satisfaction, effort, and academic performance. Computers \& Education, 80, 152-161

Hembrooke, H. y Gay, G. (2003). The laptop and the lecture: the effects of multitasking in learning environments. Journal of Computing in Higher Education, 15(1), 46-64.

Hossain, S. F. A., Nurunnabi, M., Hussain, K. y Saha, S. K. (2019). Effects of variety-seeking intention by mobile phone usage on university students' academic performance. Cogent Education, 6(1), 1574692. https:// doi.org/10.1080/2331186X.2019.1574692

Hutcheon, T. G., Lian, A. y Richard, A. (2019). The impact of a technology ban on students perceptions and performance in introduction to psychology. Teaching of Psychology, 46(1), 47-54.

Hyden, P. (2005). Teaching statistics by taking advantage of the laptop's ubiquity. New Directions for Teaching and Learning, 101, 37-42.

Ifeanyi, I. P. y Chukwuere, J. E. (2018). The impact of using smartphones on the academic performance of undergraduate students. Knowledge Management \& E-Learning: An International Journal, 10(3), 290-308.

Jacobsen, W. C. y Forste, R. (2011). The wired generation: academic and social outcomes 
of electronic media use among university students. Cyberpsychology, Behavior, and Social Networking, 14(5), 275-280.

Jamet, E., Gonthier, C., Cojean, S., Colliot, T. y Erhel, S. (2020). Does multitasking in the classroom affect learning outcomes? A naturalistic study. Computers in Human Behavior, 106. https://doi.org/10.1016/j.chb. 2020.106264

Junco, R. (2012). In-class multitasking and academic performance. Computers in Human Behavior, 28(6), 2.236-2.243.

Karamti, C. (2016). Measuring the impact of ICTs on academic performance: evidence from higher education in Tunisia. Journal of Research on Technology in Education, 48(4), 322-337.

Karpinski, A. C., Kirschner, P. A., Ozer, I., Mellott, J. A. y Ochwo, P. (2013). An exploration of social networking site use, multitasking, and academic performance among United States and European university students. Computers in Human Behavior, 29(3), 1.182-1.192.

Kay, R. H. y Lauricella, S. (2014). Investigating the benefits and challenges of using laptop computers in higher education classrooms. Canadian Journal of Learning and Technology, 40(2). https://files.eric.ed.gov/fulltext/EJ 1030425.pdf

Kennedy, D. R. (2019). The effect of banning computers on exam performance in a firstyear pathophysiology class. American Journal of Pharmaceutical Education. 83(10). https://doi.org/10.5688/ajpe7535

Kim, I., Kim, R., Kim, H., Kim, D., Han, K., Lee, P. H., Mark, G. y Lee, U. (2019). Understanding smartphone usage in college classrooms: a long-term measurement study. Computers \& Education, 141. https://doi.org/10.1016/j. compedu.2019.103611

Kolar, R. L., Sabatini, D. A. Y Fink, L. D. (2002). Laptops in the classroom: do they make a difference? Journal of Engineering Education, 91(4), 397-401.

Kraushaar, J. M. y Novak, D. C. (2010). Examining the effects of student multitasking with laptops during the lecture. Journal of Information Systems Education, 21(2), 241-251.

Kuznekoff, J. H. y Titsworth, S. (2013). The impact of mobile phone usage on student learning. Communication Education, 62(3), 233-252.

Lane, A. y Porch, M. (2002). Computer aided learning (CAL) and its impact on the performance of non-specialist accounting undergraduates. Accounting Education, 11(3), 217-233.

Lau, W. W. (2017). Effects of social media usage and social media multitasking on the academic performance of university students. Computers in Human Behavior, 68, 286-291.

Lauricella, S. y Kay, R. H. (2010). Assessing laptop use in higher education classrooms: the laptop effectiveness scale (LES). Australian Journal of Educational Technology, 26(2), 151-163.

Le Roux, D. B. y Parry, D. A. (2017). In-lecture media use and academic performance: does subject area matter? Computers in Human Behavior, 77, 86-94.

Lepp, A., Barkley, J. E. y Karpinski, A. C. (2015). The relationship between cell phone use and academic performance in a sample of US college students. Sage Open, 5(1). https://doi. org/10.1177/2158244015573169

Leyrer-Jackson, J. M. y Wilson, A. K. (2018). The associations between social-media use and academic performance among undergraduate students in biology. Journal of Biological Education, 52(2), 221-230.

Li, J., Lepp, A. y Barkley, J. E. (2015). Locus of control and cell phone use: implications for sleep quality, academic performance, and subjective well-being. Computers in Human Behavior, 52, 450-457. 
Lindroth, T. y Bergquist, M. (2010). Laptopers in an educational practice: promoting the personal learning situation. Computers \& Education, 54(2), 311-320.

Maki, R. H., Maki, W. S., Patterson, M. yWhittaker, P. D. (2000). Evaluation of a web-based introductory psychology course: I. Learning and satisfaction in on-line versus lecture courses. Behavior Research Methods, Instruments, \& Computers, 32(2), 230-239.

Martínez Rodrigo, M. E., Martínez-Cabeza Lombardo, M. Á. y Martínez-Cabeza Jiménez, J. (2019). Análisis del uso de dispositivos móviles en las aulas universitarias españolas. RLCS. Revista Latina de Comunicación Social, 74, 997-1.013.

McCabe, D. B. y Meuter, M. L. (2011). A student view of technology in the classroom: does it enhance the seven principles of good practice in undergraduate education? Journal of Marketing Education, 33(2), 149-159.

McCoy, B. (2016). Digital distractions in the classroom phase II: student classroom use of digital devices for non-class related purposes. Journal of Media Education, 4(4), 5-14.

McCreary, J. R. (2009). The laptop-free zone. Valparaiso University Law Review, 43, 1-87.

McDowall, T. y Jackling, B. (2006). The impact of computer-assisted learning on academic grades: an assessment of students' perceptions. Accounting Education: An International Journal, 15(4), 377-389.

McVay, G. J., Snyder, K. D. y Graetz, K. A. (2005). Evolution of a laptop university: a case study. British Journal of Educational Technology, 36(3), 513-524.

Montgomery, K. C. (2009). Generation Digital. MIT Press.

Mueller, P. A. y Oppenheimer, D. M. (2014). The pen is mightier than the keyboard: advantages of longhand over laptop note taking. Psychological Science, 25(6), 1.159-1.168.
Muñoz-Organero, M., Muñoz-Merino, P. J. y Kloos, C. D. (2012). Sending learning pills to mobile devices in class to enhance student performance and motivation in network services configuration courses. IEEE Transactions on Education, 55(1), 83-87.

Ng, S. F., Azlan, M. A. K., Kamal, A. N. A. y Manion, A. (2020). A quasi-experiment on using guided mobile learning interventions in ESL classrooms: time use and academic performance. Education and Information Technologies, 25(6), 4.699-4.719.

Nicol, D. J. y MacLeod, I. A. (2005). Using a shared workspace and wireless laptops to improve collaborative project learning in an engineering design class. Computers \& Education, 44(4), 459-475.

Nonis, S. A. y Hudson, G. I. (2010). Performance of college students: impact of study time and study habits. Journal of Education for Business, 85(4), 229-238.

OCDE. (2020). Spain: Overview of the Education System (EAG 2019). https://gpseducation. oecd.org/CountryProfile?primaryCountry=E SP\&treshold=10\&topic $=E O$

Palfrey, J. y Gasser, U. (2008). Born Digital. Basic Books.

Parry, D. A. y Le Roux, D. B. (2018). In-lecture media use and academic performance: investigating demographic and intentional moderators. South African Computer Journal, 30(1), 85-107.

Parry, D. A., Le Roux, D. B. y Cornelissen, L. A. (2019). Managing in-lecture media use: the feasibility and value of a split-class policy. Journal of Computing in Higher Education, 32(2), 261-281. https://doi.org/10.1007/s12 528-019-09232-z

Patterson, R. W. y Patterson, R. M. (2017). Computers and productivity: evidence from laptop use in the college classroom. Economics of Education Review, 57, 66-79.

Pechenkina, E., Laurence, D., Oates, G., Eldridge, D. y Hunter, D. (2017). Using a gamified 
mobile app to increase student engagement, retention and academic achievement. International Journal of Educational Technology in Higher Education, 14(1), 31.

Pemberton, J. R., Borrego Jr., J. y Cohen, L. M. (2006). Using interactive computer technology to enhance learning. Teaching of Psychology, 33(2), 145-147.

Poll, H. (2015). Pearson Student Mobile Device Survey: College Students. Pearson.

Prescott, W. A., Johnson, H. L., Wrobel, M. J. y Prescott, G. M. (2012). Impact of electronic device use in class on pharmacy students' academic performance. American Journal of Pharmaceutical Education, 76(9). https://doi. org/10.5688/ajpe769167

Qi, C. (2019). A double-edged sword? Exploring the impact of students' academic usage of mobile devices on technostress and academic performance. Behaviour \& Information Technology, 38(12), 1.337-1.354.

Rabl, M., Pattermann, J., Schlögl, S. y Canham, N. (2019). The use of digital devices in the university classroom: exploring and comparing students' perceptions and practices. International Workshop on Learning Technology for Education in Cloud (pp. 103-113). Springer.

Ragan, E. D., Jennings, S. R., Massey, J. D. y Doolittle, P. E. (2014). Unregulated use of laptops over time in large lecture classes. Computers \& Education, 78, 78-86.

Ramamuruthy, V. y Rao, S. (2015). Smartphones promote autonomous learning in ESL classrooms. Malaysian Online Journal of Educational Technology, 3(4), 23-35.

Rambe, P. y Bere, A. (2013). Using mobile instant messaging to leverage learner participation and transform pedagogy at a South African University of Technology. British Journal of Educational Technology, 44(4), 544-561.

Rashid, T. y Asghar, H. M. (2016). Technology use, self-directed learning, student engagement and academic performance: examining the interrelations. Computers in Human Behavior, 63, 604-612.

Ravizza, S. M., Hambrick, D. Z. y Fenn, K. M. (2014). Non-academic internet use in the classroom is negatively related to classroom learning regardless of intellectual ability. Computers \& Education, 78, 109-114.

Ravizza, S. M., Uitvlugt, M. G. y Fenn, K. M. (2017). Logged in and zoned out: how laptop internet use relates to classroom learning. Psychological Science, 28(2), 171-180.

Remón, J., Sebastián, V., Romero, E. y Arauzo, J. (2017). Effect of using smartphones as clickers and tablets as digital whiteboards on students' engagement and learning. Active Learning in Higher Education, 18(2), 173-187.

Roberts, N. y Rees, M. (2014). Student use of mobile devices in university lectures. Australasian Journal of Educational Technology, 30(4), 415-426.

Sage, K., Piazzini, M., Downey IV, J. C. y Masilela, L. (2020). Reading from print, laptop computer, and e-reader: differences and similarities for college students' learning. Journal of Research on Technology in Education, 52(4), 441-460. https://doi.org/10.10 80/15391523.2020.1713264

Salamonson, Y. y Lantz, J. (2005). Factors influencing nursing students' preference for a hybrid format delivery in a pathophysiology course. Nurse Education Today, 25(1), 9-16.

Samson, P. J. (2010). Deliberate engagement of laptops in large lecture classes to improve attentiveness and engagement. Computers in Education, 20(2), 22-37.

Sánchez, D. R., Langer, M. y Kaura, R. (2020). Gamification in the classroom: examining the impact of gamified quizzes on student learning. Computers \& Education, 144. https:// doi.org/10.1016/j.compedu.2019.103666 
Sana, F., Weston, T. y Cepeda, N. J. (2013). Laptop multitasking hinders classroom learning for both users and nearby peers. Computers \& Education, 62, 24-31.

Shuter, R., Dutta, U., Cheong, P., Chen, Y. y Shuter, J. (2018). Digital behavior of university students in India and the US: cultural values and communication technologies in the classroom. Western Journal of Communication, 82(2), 160-180.

Spies, A. R (2010). Use of laptops and other technology in the classroom. American Journal of Pharmaceutical Education, 74(8), $152 \mathrm{a}$.

Sulaiman, A. y Mohezar, S. (2006). Student success factors: identifying key predictors. Journal of Education for Business, 81(6), 328-333.

Tapscott, D. (2008). Grown Up Digital: How The Net Generation Is Changing Your World. McGraw-Hill.

Uzun, A. M. y Kilis, S. (2019). Does persistent involvement in media and technology lead to lower academic performance? Evaluating media and technology use in relation to multitasking, self-regulation and academic performance. Computers in Human Behavior, 90, 196-203.

Wakefield, J., Frawley, J. K., Tyler, J. y Dyson, L. E. (2018). The impact of an iPad-supported annotation and sharing technology on university students' learning. Computers \& Education, 122, 243-259.

Wammes, J. D., Ralph, B. C., Mills, C., Bosch, N., Duncan, T. L. y Smilek, D. (2019). Disengagement during lectures: media multitasking and mind wandering in university classrooms. Computers \& Education, 132, 76-89.

Weaver, B. E. y Nilson, L. B. (2005). Laptops in class: what are they good for? What can you do with them? New Directions for Teaching and Learning, 2005(101), 3-13.
Wei, F. Y. F., Wang, Y. K. y Fass, W. (2014). An experimental study of online chatting and notetaking techniques on college students cognitive learning from a lecture. Computers in Human Behavior, 34, 148-156.

Willemse, J. J., Jooste, K. y Bozalek, V. (2019). Experiences of undergraduate nursing students on an authentic mobile learning enactment at a higher education institution in South Africa. Nurse Education Today, 74, 69-75.

Winskel, H., Kim, T. H., Kardash, L. y Belic, I. (2019). Smartphone use and study behavior: a Korean and Australian comparison. Heliyon, 5(7). https://doi.org/10.1016/j.heliyon.2019.e02158

Witecki, G. y Nonnecke, B. (2015). Engagement in digital lecture halls: a study of student course engagement and mobile device use during lecture. Journal of Information Technology Education: Research, 14, 73-90.

Wood, E., Zivcakova, L., Gentile, P., Archer, K., De Pasquale, D. y Nosko, A. (2012). Examining the impact of off-task multi-tasking with technology on real-time classroom learning. Computers \& Education, 58(1), 365-374.

Wu, J., Mei, W. y Ugrin, J. C. (2018). Student cyberloafing in and out of the classroom in China and the relationship with student performance. Cyberpsychology, Behavior, and Social Networking, 21(3), 199-204.

Wurst, C., Smarkola, C. y Gaffney, M. A. (2008). Ubiquitous laptop usage in higher education: effects on student achievement, student satisfaction, and constructivist measures in honors and traditional classrooms. Computers \& Education, 51(4), 1.766-1.783.

Zhang, W. (2015). Learning variables, in-class laptop multitasking and academic performance: a path analysis. Computers \& Education, 81, 82-88. 


\section{ANEXO}

\section{Investigaciones empíricas sobre la relación entre el uso de los dispositivos móviles en el aula y el rendimiento académico de los estudiantes universitarios}

\begin{tabular}{|c|c|}
\hline Autores & Participantes y contexto \\
\hline $\begin{array}{l}\text { Maki et al. } \\
\text { (2000) }\end{array}$ & $\begin{array}{l}130 \text { estudiantes de un curso de Psi- } \\
\text { cología que usan ordenadores y tie- } \\
\text { nen acceso a internet y } 175 \text { que tienen } \\
\text { clase tradicional (Texas Tech. Univer- } \\
\text { sity, EE. UU.). }\end{array}$ \\
\hline
\end{tabular}

Grace-Martin 84 estudiantes de dos cursos (Comuy Gay (2001) nicación e Informática) que usan ordenadores portátiles y cuentan con acceso a internet en clase (Cornell University, EE. UU.).

\section{Principales hallazgos}

Citas Google

Scholar ${ }^{1}$

463; 22,1

(WOS-JCR) obtienen una calificación más alta que los que asisten a clase tradicional (sin acceso a internet y ordenadores) en los exámenes realizados en clase y muestran mayores ganancias en sus notas durante el curso.

Cuanto más largas son las sesiones de $134 ; 6,7$ navegación promedio que los estudiantes realizan durante la clase, más bajas son las

(WOS-JCR) calificaciones que obtienen. Las sesiones de navegación más largas son un problema para el rendimiento académico, independientemente de las características del estudiante o del curso en sí.

Los estudiantes con portátiles se desenvuelven un poco mejor en casi todos los aspectos del curso que los estudiantes que no usan portátiles, a pesar de que su promedio general de calificaciones al ingresar al curso es más bajo.

Kolar et al. (2002)
69 estudiantes de Recursos Hídricos que usan portátiles en clase (College of Engineering, University of Oklahoma, EE. UU.).
Lane y Porch

(2002)

Estudiantes de un curso introductorio en Contabilidad Financiera que usan portátiles (universidad del Reino Unido).
Las actitudes y percepciones de los estudiantes hacia el aprendizaje asistido por ordenador no tienen un efecto significativo en su rendimiento académico (calificaciones).

Los estudiantes que pueden utilizar sus portátiles para el seguimiento de las clases (y, por tanto, realizan múltiples tareas durante el desarrollo de la clase) experimentan reducciones en las pruebas de memoria relacionadas con el contenido explicado en la clase.
$70 ; 3,7$

(WOS-ESCl, SJR) $657 ; 36,5$

(WOS-JCR) el seguimiento de las clases (universidad del noreste de EE. UU.). 


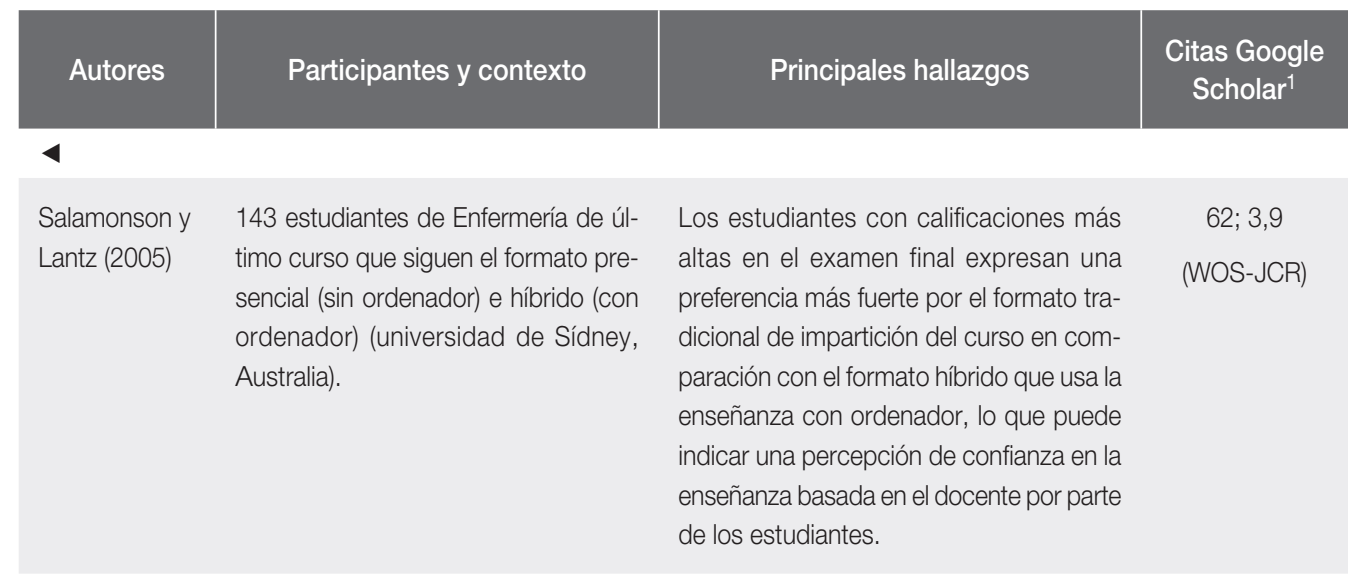

McDowall 280 estudiantes de segundo año y Jackling de Contabilidad de una universidad (2006) australiana que utilizan paquetes de "aprendizaje asistido por ordenador" (universidad de Australia).

Pemberton

377 estudiantes de ocho clases de et al. (2006) Psicología que utilizan en clase una herramienta de enseñanza interactiva -LearnStar- (Texas Tech. University, EE. UU.).

Las percepciones positivas de la utilidad del aprendizaje asistido por ordenador (uso de ordenadores para la enseñanza) afectan significativamente el rendimiento académico. Los estudiantes internacionales tienen un desempeño (notas) significativamente peor que los estudiantes locales.

Los estudiantes que usan LearnStar (herramienta basada en el uso del ordenador en clase) no obtienen calificaciones significativamente diferentes respecto a los estudiantes de las clases (tradicionales) que no la utilizan. Por tanto, la introducción de esta herramienta puede no ser una forma efectiva de mejorar el rendimiento académico.

El uso de los portátiles interfiere en la capacidad de los estudiantes para prestar atención y comprender el material explicado en clase, lo que, a su vez, da como resultado puntuaciones más bajas en las pruebas. Cuantos más estudiantes usan sus portátiles en clase, menor es su desempeño. El nivel de uso del portátil está relacionado de manera negativa y significativa con varias medidas de aprendizaje de los estudiantes, incluido el rendimiento general del curso.

$64 ; 4,3$

(WOS-ESCl, SJR)
53; 3,5

(WOS-JCR)

$958 ; 73,7$

(WOS-JCR) 


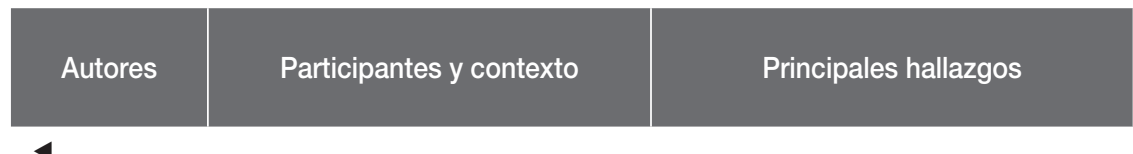

Citas Google

Scholar ${ }^{1}$

Wurst et al. (2008)

87 estudiantes de una escuela de negocios (tres años) que pueden utilizar portátiles en sus clases (universidad de EE. UU.).
Carle et al. (2009)

Fox et al. (2009)

Ellis et al. (2010)
El uso de los portátiles por los estudiantes y el cuerpo docente no aumenta el nivel de actividades constructivistas en las aulas. El uso de los portátiles en clase no mejora estadísticamente el rendimiento de los estudiantes según se refleja en el promedio de sus calificaciones. Los estudiantes que usan portátiles en clase muestran un nivel de satisfacción estadísticamente menor con su educación en comparación con los estudiantes que no los usan.
259; 19,9

(WOS-JCR)

\section{(}

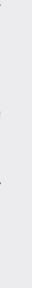
usa la mensajería instantánea, más bajas son las puntuaciones en comprensión de la lectura y más bajo es el promedio de las calificaciones obtenidas.
25 estudiantes de dos secciones de Métodos de Investigación de Psicología que usan dispositivos de audio portátiles en clase (por ejemplo, iPods) (universidad del sureste de EE. UU.).
Los estudiantes que utilizaban en sus clases los dispositivos de audio portátiles consiguen mejoras estadísticamente significativas en su participación en clase, asi como un mejor rendimiento académico.
69 estudiantes de un curso de Intro-

Los estudiantes que envían mensajes instantáneos mientras realizan la tarea de lectura en clase tardan mucho más tiempo en completar la tarea. Los análisis adicionales revelan que cuanto más tiempo se

$108 ; 9,0$

(WOS-JCR)

$278 ; 23,2$

(WOS-JCR) mensajería instantánea en clase me diante el uso de portátiles (universidad de EE. UU.).
Las calificaciones de los estudiantes que usan los teléfonos celulares son significativamente más bajas que las calificaciones de los exámenes de los estudiantes que no los utilizan. Independientemente del género o del promedio de las calificaciones, el rendimiento académico es más bajo cuando la multitarea tiene lugar en un entorno de aprendizaje.
$205 ; 18,6$

(WOS-JCR) sus teléfonos celulares en clase (universidad del sureste de EE. UU.). $275 ; 25$
El uso de tablets durante la impartición de las clases, para crear una red de aprendi- zaje interactive, muestra un efecto positivo
Enríquez
62 estudiantes de un curso introductorio
(2010) de Análisis de Circuitos que usan tablets y una aplicación de software en clase

(SJR) 


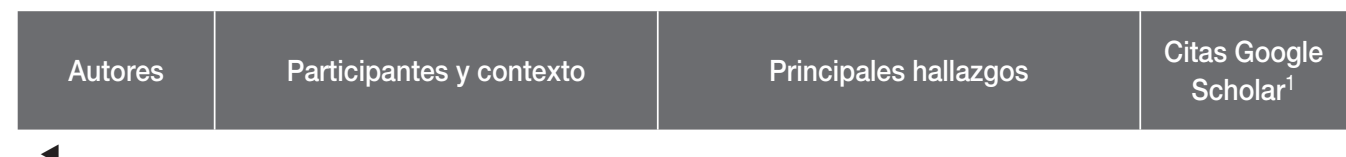

$\begin{array}{ll}\text { Enríquez } & \text { (Escuela NetSupport, Cañada College } \\ \text { (2010) (cont.) } & {[16] \text { y San Francisco State University }} \\ & {[46], \text { EE. UU.). }}\end{array}$

Kraushaar y

Novak (2010)
97 estudiantes de tres secciones diferentes de un curso obligatorio en Sistemas de Gestión de la Información que pueden usar portátiles en clase (The University of Vermont, EE. UU.).
Jacobsen y

Forste (2011)

1.026 estudiantes de primer año de varias titulaciones que usan diversos dispositivos electrónicos en sus actividades académicas (universidad privada de EE. UU.). y estadísticamente significativo en el desempeño (notas) de los estudiantes. Además, los resultados de las encuestas a los estudiantes muestran que su percepción sobre los efectos de este ambiente de clase en su experiencia de aprendizaje es abrumadoramente positiva.
Los estudiantes se involucran en un comportamiento sustancial de multitarea con sus portátiles y tienen aplicaciones de software abiertas y activas, no relacionadas con el curso, aproximadamente, el $42 \%$ del tiempo de la clase. Existe una relación inversa estadísticamente significativa entre el comportamiento de distracción versus el comportamiento productivo de realización de múltiples tareas durante las clases y las calificaciones obtenidas.

El uso de dispositivos electrónicos (por ejemplo, portátiles, móviles, etc.) se relaciona negativamente con las calificaciones. En torno a dos tercios de los estudiantes usan dispositivos electrónicos mientras están en clase, estudian o realizan tareas. Esta multitarea probablemente aumenta la distracción, algo que puede ser perjudicial para el rendimiento académico.

Las calificaciones obtenidas en el examen no son significativamente diferentes para los estudiantes que no utilizan los portátiles, tanto si están en la zona en la que se encuentra permitido su uso como en la que no, lo que indica que el uso de portátiles en clase no afecta al rendimiento general de los estudiantes de los alrededores. Sin embargo, quienes no utilizan los portátiles en clase obtienen una nota significativamente más alta que los que sí los utilizan.
275; 25

(SJR)

$403 ; 36,6$

(SJR)
Aguilar-Roca

et al. (2012)
Más de 400 estudiantes de un curso de Biología separados en zonas d tro del aula en los que se puede no se puede usar el portátil (universdad de Canadá).
$134 ; 14,9$

(WOS-JCR) 


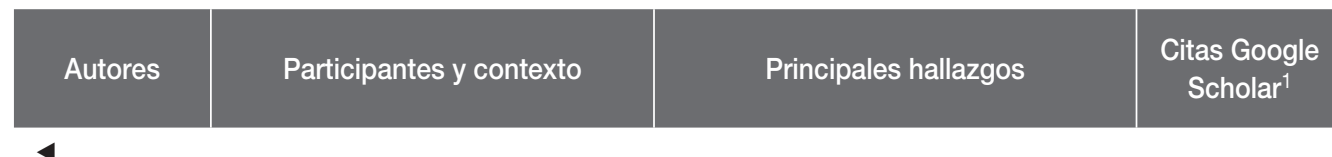

Froese et al. Estudio 1: 693 estudiantes de varias (2012) universidades de EE. UU. que llevan sus móviles a clase.

Estudio 2: 40 estudiantes de una universidad de EE. UU. que llevan sus móviles a clase.
Estudio 1: los estudiantes prevén obtener una nota significativamente mejor si no envían mensajes de texto con sus móviles durante la clase.

Estudio 2: las notas de los exámenes son significativamente más bajas cuando los estudiantes envían mensajes de texto que cuando no lo hacen. La diferencia en las puntuaciones representa una disminución del $27 \%$ durante el envío de mensajes de texto con respecto al rendimiento sin enviar mensajes de texto.

Junco (2012) 1.774 estudiantes de varias titulaciones que usan en clase móviles y portátiles (universidad pública del noreste de EE. UU.).
El uso de los dispositivos móviles durante la clase vinculados a las redes sociales (Facebook y los mensajes de texto) se relacionan negativamente con la media de las calificaciones obtenidas por los estudiantes. Otras actividades realizadas durante la clase con tales dispositivos (como, por ejemplo, búsquedas o envíos de e-mails) no tienen ningún efecto en la media de las calificaciones.
$167 ; 18,6$

(SJR)

$382 ; 42,4$

(WOS-JCR)

\begin{abstract}
La asistencia media a clase y la motivación de los estudiantes mejora cuando se usan las píldoras de aprendizaje contextualizadas (esto es, los estudiantes usan los dispositivos móviles en clase para realizar ejercicios cortos). Las notas medias de este grupo de estudiantes son significativamente mejores que la de los estudiantes que no las usan.

Muñoz-Orga- 170 estudiantes de la Escuela Politecnero et al. nica Superior que pueden usar dispo (2012) Sarlos III de Madrid).
\end{abstract}

Cuando se combinan los estudiantes P2 y P3, el uso de dispositivos electrónicos (como portátiles, móviles y tablets) no se asocia con las calificaciones. Las calificaciones no se ven afectadas entre los estudiantes P3, pero los estudiantes P2 obtienen mejores notas si se abstienen de usar dispositivos electrónicos en clase.
$32 ; 3,6$

(WOS-JCR)

$14 ; 1,6$

(WOS-JCR)

\section{tercer año (P3) que pueden usar dis- positivos móviles en clase (universidad de EE. UU.). \\ (2012) macoterapéutica de segundo (P2) y}

140 estudiantes de un curso de Far- 


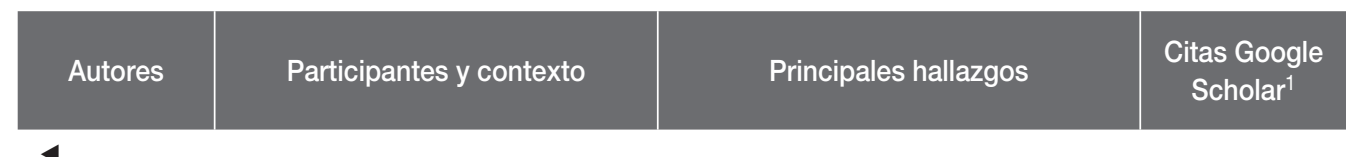

Wood et al. 145 estudiantes de los cursos de Mé(2012) todos de Investigación y Estadística de segundo año que usan dispositivos móviles en clase (universidad de Canadá).

\section{Clayson y}

Haley (2013)
298 estudiantes de Marketing que usan portátiles en clase (2 universidades de EE. UU.).
Karpinski et al. 451 estudiantes de EE. UU. y 406 (2013) de Europa que consultan páginas de redes sociales en clase a través de sus dispositivos móviles.
En general, los estudiantes que no usan ningún tipo de dispositivo móvil en clase para realizar distintas tareas obtienen mejores resultados académicos que los estudiantes que los utilizan para llevar algún tipo de tarea.

A pesar de que los estudiantes creen que pueden seguir la clase mientras mandan mensajes de texto, los que lo hacen obtienen calificaciones más bajas. La frecuencia del envío de mensajes no está relacionada con la nota media final.

La relación negativa entre el uso de redes sociales y las notas medias obtenidas está moderada por la multitarea solo en la muestra de EE. UU. Los estudiantes estadounidenses son más capaces de realizar múltiples tareas en comparación con los estudiantes europeos. Los estudiantes europeos parecen ser menos propensos a lo que se denomina «multitarea disruptiva».

Los estudiantes que no usan sus móviles escriben un $62 \%$ más de información en sus apuntes, toman notas más detalladas, son capaces de recordar información más detallada de la clase y obtienen calificaciones más altas que aquellos estudiantes que utilizan activamente sus móviles durante la clase.

Los estudiantes que realizan múltiples tareas (a raíz del uso del portátil) tienen una comprensión reducida del material impartido en clase y obtienen una nota más baja en el examen en comparación con los que no realizan múltiples tareas (no usan el portátil). Utilizar el ordenador o estar sentado cerca de alguien que lo utiliza obstaculiza el aprendizaje.
630; 70

(WOS-JCR)

$86 ; 10,8$

(WOS-ESCl, SJR)

$359 ; 44,9$

(WOS-JCR)

$382 ; 29,4$

(WOS-JCR)

$758 ; 94,8$

(WOS-JCR) sus portátiles en clase (universidad de Canadá).

$\begin{array}{lll}\text { Sana et al. } & 44 \text { estudiantes de un curso introduc- } & \text { Los estudiantes que realizan múltiples ta- } \\ \text { (2013) } & \text { torio de Psicología que pueden usar } & \text { reas (a raíz del uso del portátil) tienen una } \\ \text { sus portátiles en clase (universidad de } & \text { comprensión reducida del material impar- } \\ & \text { Canadá). } & \text { tido en clase y obtienen una nota más baja } \\ & \text { en el examen en comparación con los que } \\ & \text { no realizan múltiples tareas (no usan el por- } \\ & \text { tátil). Utilizar el ordenador o estar sentado } \\ & \text { cerca de alguien que lo utiliza obstaculiza } \\ & \text { el aprendizaje. }\end{array}$




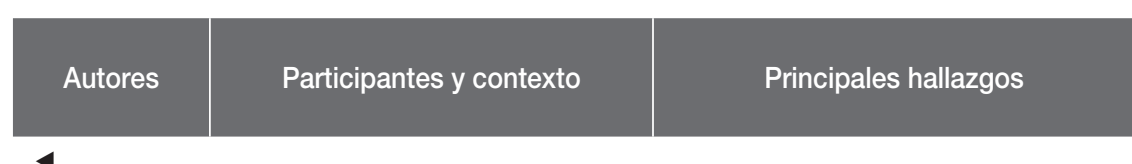

Citas Google

Scholar ${ }^{1}$

Gaudreau et al. (2014)
Estudio 1: 1.129 estudiantes de varias materias que usan portátiles en clase (universidad pública de Ontario, Canadá) (transversal).

Estudio 2: 88 estudiantes que usan portátiles en clase (universidad pública de Ontario, Canadá) (Iongitudinal).
MuelleryOppen- Estudio 1: 67 estudiantes de varias heimer (2014) EE. UU.)

Estudios 2 y 3: 151 y 109 alumnos/as respectivamente que usan portátiles en clase para tomar notas (Universidad de California, EE.UU.).
Estudio 1: el uso de los portátiles para actividades no relacionadas con la clase se vinculan negativamente tanto con el rendimiento académico como con la satisfacción. Su uso para actividades de la clase se relaciona significativamente con la satisfacción y débilmente con el rendimiento académico.

Estudio 2: en general, el uso de los portátiles con actividades de clase se relaciona con un promedio de calificaciones más bajo, incluso tras controlar otros factores. En ambos estudios, el uso de portátiles para actividades de clase no está relacionado con el éxito académico.

En los tres estudios se obtiene que los estudiantes que toman notas en portátiles se desenvuelven peor en preguntas conceptuales que los estudiantes que toman notas a mano. Si bien tomar más notas puede ser beneficioso, la tendencia a transcribir lo explicado de quienes toman notas con portátiles en clase es perjudicial para el aprendizaje dada su menor capacidad de procesamiento de la información explicada.

Ravizza et al

170 estudiantes de una clase de Intro(2014) ducción a la Psicología que usan dispositivos móviles en clase para acceder a internet (universidad de EE. UU.).
Las tasas más altas de uso de internet durante la clase están asociadas con calificaciones más bajas en las pruebas realizadas y las creencias de los estudiantes sobre esta relación no reflejan su capacidad para realizar múltiples tareas de manera efectiva, por lo que su uso genera distracción que disminuye el aprendizaje.

Los estudiantes que realizan frecuentemente múltiples tareas en clase tienen notas medias finales más bajas. El envío de mensajes de texto es una actividad dominante tanto cuando se asiste a clase como cuando se realizan tareas en casa.
$122 ; 17,4$

(WOS-JCR)

$1.258 ; 179,7$

(WOS-JCR)

$247 ; 35,3$

(WOS-JCR)

$130 ; 21,7$

(WOS-JCR) $\begin{array}{ll}\text { Bellur et al. } & 361 \text { estudiantes de un curso de Comu- } \\ \text { (2015) } & \text { nicación que pueden usar sus móviles }\end{array}$ en clase (universidad de EE. UU.). 


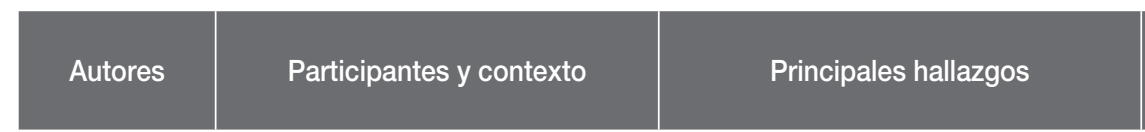

Brooks (2015) 209 estudiantes de un curso de Sistemas de Información que usan dispositivos móviles para acceder a redes sociales en clase (universidad del oeste de EE.UU.).

Downs et al.
$\begin{array}{ll}204 \text { estudiantes de Comunicación que } \\ \text { (2015) }\end{array}$
usan portátiles en clase (universidad
del medio oeste, EE. UU.).

Lepp et al. $\quad 536$ estudiantes de varias especiali(2015) dades que usan sus móviles en clase (universidad de EE. UU.).

Li et al. (2015) 516 estudiantes de una gran universidad pública que usan móviles (universidad del medio oeste de EE. UU.).

Zhang (2015) 177 estudiantes de Métodos de Investigación que usan portátiles en clase (universidad de Singapur).

Green (2016)
Un mayor tiempo de uso personal de las redes sociales en clase conduce a un menor rendimiento en la realización de las tareas (y, por tanto, puede llevar a peores resultados académicos), así como a niveles más altos de tecnoestrés y menor felicidad de los estudiantes que las utilizan.

Los estudiantes que obtienen las mejores calificaciones son aquellos que no utilizan los portátiles durante la clase dado que es más probable que se distraigan accediendo a las redes sociales.

Un mayor uso de los móviles en clase se relaciona de manera negativa y significativa con el rendimiento académico (nota media final) de aquellos estudiantes que los utilizan.

El uso de móviles en clase y mientras se estudia se relaciona negativamente con el rendimiento académico. Un mayor «locus» de control interno puede mitigar algunos de los resultados negativos asociados con el uso frecuente de los móviles; a la inversa, en estudiantes con un mayor "locus" de control externo, los efectos negativos pueden exacerbarse.

La realización de multitareas en clase debido al uso del portátil se asocia negativamente con las calificaciones obtenidas. La relación negativa entre la realización de multitareas y las notas solo es significativa en el contexto de la clase, pero no es significativa en otros lugares, como salas de tutoría, bibliotecas u hogares de los estudiantes.

El uso de dispositivos móviles en clase (es decir, el uso de diferentes apps de enseñanza) ayuda a mejorar la nota de los Citas Google Scholar ${ }^{1}$ $352 ; 58,7$ (WOS-JCR) $31 ; 5,2$ (WOS-JCR) $366 ; 61$ (WOS-JCR) $144 ; 24$ (WOS-JCR) exámenes en más del $8 \%$, en promedio, en comparación con los estudiantes que no hacen uso de los mismos.
$77 ; 12,8$

(WOS-JCR) (Lander University, EE. UU.).

69 estudiantes, en cuatro secciones de Principios de Macroeconomía, que 


\section{\begin{tabular}{c|r} 
Autores & Participantes y contexto \\
\hline & \\
Karamti (2016) & 187 estudiantes que usan ordenadores
\end{tabular} (universidad de Túnez).}

\section{Principales hallazgos}

\section{Citas Google}

Scholar ${ }^{1}$

$51 ; 10,2$

(WOS-JCR) entre el uso intensivo de los ordenadores (en clase y fuera de ella) y el rendimiento académico (calificaciones).

\begin{abstract}
Rashid y 761 alumnas que utilizan diferentes Asghar (2016) dispositivos móviles (universidad privada en Arabia Saudi).

Carter et al. $\quad 50$ aulas y 726 estudiantes de un (2017) curso de Introducción a la Economía que pueden utilizar portátiles y tablets en clase (Academia Militar de los Estados Unidos).
\end{abstract}

El uso de la tecnología tiene una relación positiva directa con la participación de las estudiantes y el aprendizaje autodirigido, pero no se encuentra un efecto directo significativo entre el uso de la tecnología y la nota media obtenida.
Las notas medias de los exámenes finales entre los estudiantes asignados a las aulas que permiten dispositivos móviles son 0,18 desviaciones estándar más bajas que las notas medias de los exámenes finales de los estudiantes en las aulas en las que está prohibido el uso de tales dispositivos. con fines no académicos (uso de videojuegos en particular) y la realización de multitareas en las redes sociales predice de manera negativa y significativa el rendimiento académico.
$349 ; 69,8$

(JCR)

$143 ; 35,8$

(WOS-JCR)
El uso de las redes sociales con fines académicos no es un predictor significativo del rendimiento académico (nota media final), mientras que el uso de las redes sociales

$275 ; 68,8$

(WOS-JCR) pósitos académicos (universidad de Hong Kong).
La influencia del uso de dispositivos móviles sobre el rendimiento académico en general y en clase difiere según la materia. Hay una correlación negativa significativa entre el uso de tales dispositivos en clase y el rendimiento académico en estudiantes de Artes y Ciencias Sociales. No se observa el mismo patrón para los estudiantes de las facultades de Ingeniería, Ciencias Económicas y de Gestión y Ciencias Médicas y de la Salud.
$32 ; 8$

(WOS-JCR) 


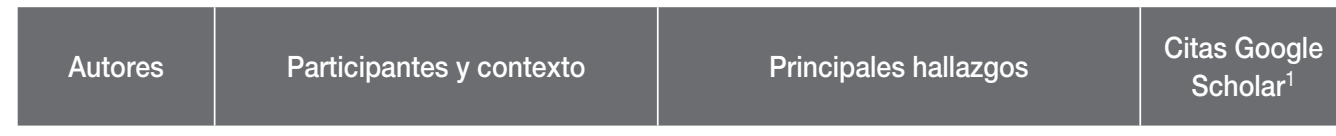

Patterson y $\quad 5.571$ estudiantes de Artes LiberaPatterson les, en el transcurso de seis semes(2017) tres entre 2013 y 2015, que pueden usar sus portátiles en clase (universidad de EE. UU.).
El uso de portátiles en las clases tiene un impacto negativo y significativo en el rendimiento del curso de los estudiantes en una escala de entre 0,14-0,37 puntos de calificación o 0,17-0,46 desviaciones estándar.
Pechenkina

et al. (2017)
711 estudiantes de Contabilidad y Ciencias de primer año que usan una app de aprendizaje gamificado en clase (universidad de Australia).
Ravizza et al. (2017)

Remón et al. (2017)
84 estudiantes de una clase de Introducción a la Psicología que pueden usar portátiles en clase para acceder a internet (Michigan State University, EE. UU.).
Las tasas de retención y el rendimiento académico de los estudiantes aumentan tras la introducción de la app móvil. Existe una correlación positiva entre las puntuaciones más altas dadas por los estudiantes a la app y el logro de calificaciones académicas más altas.

El uso no académico de internet es común entre los estudiantes que usan sus portátiles en clase y está inversamente relacionado con las notas obtenidas. Esta relación se mantiene después de considerar la motivación, el interés y la inteligencia. El uso de internet relacionado con lo que se está tratando en la clase no se asocia con un beneficio en las notas.

Se obtienen mejores resultados cuando se hace uso de lecciones interactivas con tablets que con el aprendizaje tradicional (PowerPoint incluido). El uso de procedimientos interactivos en clase aumenta la participación de los estudiantes. Además, los estudiantes son muy receptivos al uso de móviles como medio de estudio.

El uso de la tecnología de dispositivos móviles (es decir, la app utilizada) tiene una influencia positiva y significativa en los resultados del aprendizaje de los estudiantes (mejores notas en el examen test realizado).

(WOS-JCR)

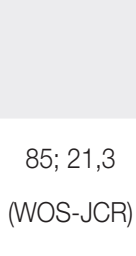

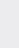




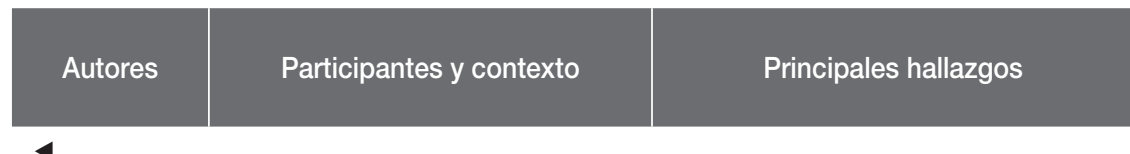

Citas Google

Scholar $^{1}$

Demirbilek y

Talan (2018)
122 estudiantes de la Facultad de Educación y de la Escuela Superior Profesional de Servicios de Salud que usan dispositivos móviles en clase (universidad de Turquía).
Elliott-Dorans (2018)

152 estudiantes de un curso de introducción a la Política Estadounidense en dos semestres que pueden usar portátiles en clase (universidad pública del medio este de EE. UU.).
Cuando a los estudiantes se les da la oportunidad de realizar múltiples tareas no relacionadas con la clase usando dispositivos móviles para escribir/enviar SMS, ver perfiles de Facebook, leer noticias/ver mensajes multimedia compartidos, etc., durante la clase, sus calificaciones empeoran en comparación con los estudiantes que no los usan.

Cuando a los estudiantes se les prohíbe usar portátiles durante la clase, es menos probable que asistan a la misma, realizan peor las tareas escritas y, durante un semestre, obtienen notas más bajas en el examen final. Los estudiantes no penalizan al docente por implementar una política restrictiva de uso de portátiles en clase.
Felisoni y

Godoi (2018)
43 estudiantes de Administración de Empresas que usan diariamente sus móviles (Fundação Getúlio Vargas/ Escuela de Negocios de São Paulo, Brasil).
Cada 100 minutos (en promedio) que se dedican diariamente al uso del móvil supone una bajada en la posición de un estudiante de 6,3 puntos en la clasificación que establece la escuela a partir de sus notas, en un rango que oscila de 0 a 98,4. Además, si se considera su uso solo durante el tiempo de clase, el efecto es casi el doble.

$\begin{array}{ll}\text { Giunchiglia } & 72 \text { estudiantes que usan redes socia- } \\ \text { et al. (2018) } & \text { les en móviles (University of Trento, } \\ & \text { Italia). }\end{array}$

\section{Giunchiglia} Italia).
El uso de aplicaciones de redes sociales durante las actividades académicas (en términos de sesiones y duración) muestra una asociación negativa con el rendimiento académico de los estudiantes (por ejemplo, con su nota media final).
Ifeanyi y $\quad 375$ estudiantes de varias facultades

Chukwuere (2018) que usan móviles (North-West University, Sudáfrica).
El uso de móviles por parte de los estudiantes les beneficia académicamente porque aumenta su rendimiento, aunque no de una manera enorme, pero lo incrementa de todos modos. El uso eficaz de los móviles aporta más ventajas que desventajas a la vida de los estudiantes examinados.

$31 ; 10,3$

(WOS-JCR)

$21 ; 7$

(WOS-JCR)

$120 ; 40$

(WOS-JCR)
$120 ; 40$

(WOS-JCR)

29; 9,7

(WOS-JCR) 


\begin{tabular}{|c|c|c|c|}
\hline Autores & Participantes y contexto & Principales hallazgos & $\begin{array}{c}\text { Citas Google } \\
\text { Scholar }^{1}\end{array}$ \\
\hline \multicolumn{4}{|l|}{4} \\
\hline $\begin{array}{l}\text { Leyrer-Jack- } \\
\text { son y Wilson } \\
\text { (2018) }\end{array}$ & 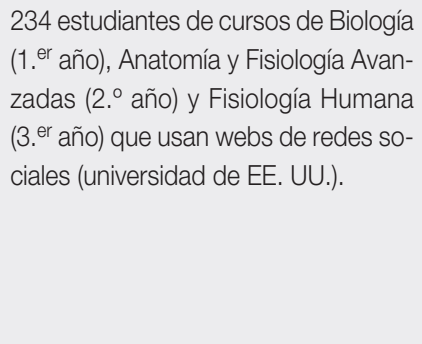 & $\begin{array}{l}\text { La nota final media se correlaciona nega- } \\
\text { tivamente con el número de sitios web de } \\
\text { redes sociales a los que se suscriben los } \\
\text { estudiantes. La adicción a las redes so- } \\
\text { ciales puede tener efectos negativos en } \\
\text { los hábitos de estudio y en el rendimiento } \\
\text { académico de los estudiantes, particular- } \\
\text { mente en aquellos que se encuentran en el } \\
\text { campo de las ciencias biológicas. }\end{array}$ & $\begin{array}{c}\text { 31; 10,3 } \\
\text { (WOS-JCR) }\end{array}$ \\
\hline
\end{tabular}

Le Roux y 120 estudiantes que usan dispositiParry (2018) vos móviles en clase (universidad de Sudáfrica).
Hay una correlación negativa significativa entre el número de veces que se usan los dispositivos móviles en las clases y el rendimiento académico. Este efecto es independiente de los factores demográficos individuales y de la intención con la que se utilizan tales dispositivos.
$11 ; 3,7$

$(\mathrm{SJR})$

$\begin{array}{ll}\text { Wu Mei y } & 1.050 \text { estudiantes de varios cursos } \\ \text { Ugrin (2018) } & \begin{array}{l}\text { que pueden usar sus dispositivos mó- } \\ \text { viles en clase (universidad de China). }\end{array}\end{array}$

\section{Wu Mei y} viles en clase (universidad de China).
Existe una relación negativa entre el uso de internet para propósitos no académicos en el aula y la nota media final. Aunque esta distracción es dañina en el aula, puede tener efectos positivos cuando se realiza con moderación fuera del aula como medio de recuperación del esfuerzo.
$40 ; 13,3$

(WOS-JCR)

Un aumento de una desviación estándar en el uso diario de móviles produce una disminución en las notas promedio de los exámenes de aproximadamente un punto (de 20). La asociación negativa entre el uso de móviles y las calificaciones de los exámenes es más clara para los estudiantes cuyos padres tienen estudios superiores, están divorciados o gozan de buena salud,
32; 16

(WOS-JCR)
Dunn y Ken- nedy (2019) (universidad del Reino Unido).
524 estudiantes de Psicología que usan technology enhanced learning

Baert et al. (2019)
696 estudiantes de primer año que usan smartphones en clase (Ghent University y University of Antwerp, ambas en Bélgica).
El compromiso de los estudiantes con la nueva tecnología más que su uso es lo que ayuda a predecir las notas obtenidas.
70; 35

(WOS-JCR) 


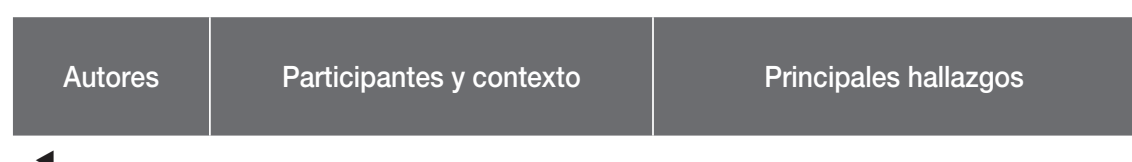

Citas Google

Scholar $^{1}$

Glass y Kang 118 estudiantes de un curso de Psi(2019)

cología Cognitiva de nivel superior que usan dispositivos móviles en clase (universidad estatal emblemática en EE. UU.).
Hossain et al. 311 estudiantes de cuatro universida(2019) des de Bangladés que usan móviles.
Cuando se permite el uso de dispositivos móviles en clase, las notas obtenidas en un examen final son peores tanto en el grupo de estudiantes que lleva tales dispositivos a clase y los usa activamente como en el grupo que los lleva pero no los usa en comparación con las obtenidas por los estudiantes del grupo de control (estudiantes que no llevan tales dispositivos a clase).

El uso de los móviles con propósitos de búsqueda de información tiene una fuerte relación positiva con los resultados académicos, mientras que su uso continuo para acceder a redes sociales presenta un efecto negativo.
El rendimiento de los estudiantes en los exámenes y en el curso mejora dentro del aula sin portátiles, especialmente para los estudiantes situados en la parte inferior de la escala de calificaciones. La nota media del examen aumenta un $3 \%$ en el aula cuando no se usan los portátiles.
56; 28

(WOS-JCR)
$39 ; 19,5$

(WOS-ESCl, SJR) $2 ; 1$

(WOS-JCR) clase (Western New England University, EE. UU.).

Los estudiantes usan sus móviles durante más del $25 \%$ de la duración efectiva de la clase y las distracciones ocurren cada 3 $27 ; 13,5$

Kim et al. $\quad 84$ estudiantes de primer año que usan (2019) smartphones en clase (universidad de Corea del Sur). a 4 minutos durante más de 1 minuto. E uso del móvil en clase se correlaciona negativamente con las calificaciones de los estudiantes.

\begin{abstract}
Hutcheon
et al. (2019)

69 estudiantes de Introducción a la Psicología que pueden usar dispositivos móviles (Bard College, EE. UU.).
\end{abstract}

Qi (2019) 208 estudiantes de Sistemas de Gestión de la Información que usan distintos dispositivos móviles (universidad de China).
No hay diferencias en las calificaciones finales de los estudiantes que no usan los dispositivos móviles en clase respecto a los que sí los utilizan.
El uso académico de los dispositivos móviles no provoca tecnoestrés y ayuda a mejorar el rendimiento académico (nota $23 ; 11.5$ (WOS-JCR)
$8 ; 4$

(WOS-JCR) media final acumulada). 


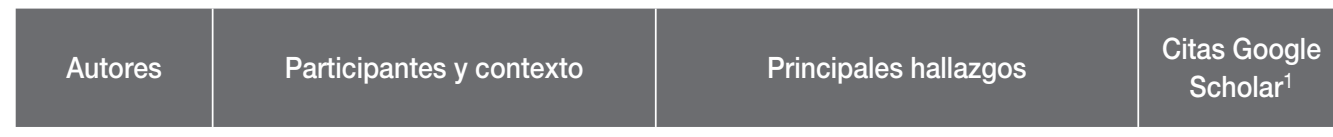

Uzun \& Kilis (2019)
631 estudiantes de Facultad de Educación que usan dispositivos móviles (Afyon Kocatepe University, Turquía).
La nota media final de los estudiantes muestra una asociación negativa significativa débil tanto con las preferencias de multitarea como con el uso de dispositivos móviles. Las variables demográficas (incluido el sexo, la edad y el año de estudio) son predictores significativos del rendimiento académico. Después de controlar estas variables, el uso de dispositivos móviles contribuye de manera negativa y significativa al rendimiento académico.

Existe una relación negativa entre el uso promedio de móviles y la nota final media para los estudiantes australianos. Por el contrario, para los estudiantes coreanos, no existe una relación significativa.
$27 ; 13,5$

(WOS-JCR)
Winskel et al. 119 estudiantes surcoreanos (Kyung(2019) nam University) y 270 estudian universitarios australianos (Southern Cross University) que usan móviles.
El uso de dispositivos móviles en clase para tomar apuntes no tiene un impacto estadísticamente significativo en las calificaciones; el problema probablemente radica en la elección de uso que se hace de los mismos.

Los estudiantes que siguen actividades lingüísticas guiadas utilizando dispositivos móviles tienen niveles significativamente más altos de rendimiento lingüístico. Los que pasan más tiempo usando dispositivos móviles para aprender el idioma no obtienen calificaciones más altas en comparación con los que pasan un tiempo mínimo.

No existe evidencia concluyente de que el uso de dispositivos móviles durante la clase mejore o empeore las calificaciones obtenidas por los estudiantes durante el semestre considerado.
$25 ; 12,5$

(SJR)

$$
5 ; 5
$$

(WOS-JCR)
Parry et al. (2020)
67 estudiantes de tres facultades que puedes usar dispositivos móviles en clase (universidad de Sudáfrica).

\begin{abstract}
usan dispositivos móviles para sus lecciones (universidad de Malasia).

$\mathrm{Ng}$ et al.
\end{abstract}

(1)

\begin{tabular}{ll}
$\begin{array}{l}\text { Artz et al. } \\
(2020)\end{array}$ & $\begin{array}{l}230 \text { estudiantes de Principios de Micro- } \\
\text { economía que usan dispositivos móvi- } \\
\text { les en clase (universidad de EE. UU.). }\end{array}$ \\
\hline $\begin{array}{l}\text { Ng et al. } \\
(2020)\end{array}$ & $\begin{array}{l}405 \text { estudiantes de un curso de Inglés } \\
\text { para Ciencias de dos facultades que } \\
\text { usan dispositivos móviles para sus lec- } \\
\text { ciones (universidad de Malasia). }\end{array}$
\end{tabular}

